\title{
Electron cyclotron resonance charge breeder plasma parameters from the modeling of $1+$ beam stopping
}

\author{
A. Annaluru $0^{*}$ \\ GANIL, CEA/DSM-CNRS/IN2P3, 14076 Caen Cedex 05, France \\ and Pantechnik, 13 Rue de la Résistance, 14400 Bayeux, France \\ P. Delahaye $\odot$, M. Dubois, and L. Maunoury 1 \\ GANIL, CEA/DSM-CNRS/IN2P3, 14076 Caen Cedex 05, France
}

(Received 11 March 2021; accepted 10 June 2021; published 30 July 2021)

\begin{abstract}
As a part of SPIRAL1 upgrade, several experiments were carried out to understand the transport of $1+$ (monocharged) ion beam through the SP1 ECR charge breeder ion source and to investigate the physical processes involved in charge breeding. Ion beam transport simulations were performed to reproduce the trends of the experimental results (charge breeding efficiency versus $1+$ beam injection energy $\Delta \mathrm{V}$ ) by transporting the $1+$ beam through a potential map that reflects the presence of the ECR (electron cyclotron resonance) plasma. The role of Coulomb collisions in the capture of $1+$ beam leads to a necessary detailed analysis for an accurate description of the charge breeding process. This was done by using a full six-dimensional (6D) phase space Monte Carlo charge breeding (MCBC) code. MCBC models Coulomb collisions of the injected 1+ beam in an ECR plasma and atomic processes (ionization and charge exchange). A simplified background ECR plasma model is implemented in the code to reproduce the trends of three different experiments (interaction of $\mathrm{Na}^{1+}$ with He plasma, $\mathrm{K}^{1+}$ with $\mathrm{He}$ plasma and $\mathrm{K}^{1+}$ with $\mathrm{O}_{2}$ plasma). The model is able to reproduce the low charge state $(1+$ and $2+)$ experimental trends by tuning each plasma parameter (plasma density, plasma ion temperature and electron temperature) independently. The estimated plasma parameters obtained from each case are presented and their contribution in charge breeding process are discussed.
\end{abstract}

DOI: 10.1103/PhysRevAccelBeams.24.073403

\section{INTRODUCTION}

GANIL, Grand Accélérateur National d'Ions Lourds, is one of the advanced accelerator laboratories to use ISOL (isotope separation on line) [1] as well as high energy projectile fragmentation methods for exploring the structure of exotic nuclei far from stability with intense stable and radioactive ion beams (RIBs) [2]. A primary ion beam accelerated by GANIL cyclotrons producing a wide range of radioactive nuclei by impinging on a hot production target coupled with a ECRIS (electron cyclotron resonance ion source) located in the SPIRAL1 facility. The radioactive atoms released from the target effuse into the ion source (ECRIS-NANOGAN III) [3,4] via a cold transfer tube. The radioactive atoms are then ionized and extracted as multicharged radioactive ion beam. During the past decades, the SPIRAL1 facility in GANIL has been

\footnotetext{
*arun.annaluru@pantechnik.com
}

Published by the American Physical Society under the terms of the Creative Commons Attribution 4.0 International license. Further distribution of this work must maintain attribution to the author(s) and the published article's title, journal citation, and DOI. producing and delivering around 35 intense radioactive ion beams of gaseous elements for physics experiments. In 2012, a major upgrade has been undertaken [5] to extend the production capabilities to a number of condensable elements [6]. To this intent, a FEBIAD (forced electron beam induced arc discharge) TISS (target ion source system) has been chosen for the production of monocharged condensable RIBs with high yields [7,8]. 1+ RIBs from TISS have to match the injection characteristics of the CIME cyclotron [9]. Therefore, a charge breeder has been put into practice between the TISS and the CIME cyclotron to increase the charge states of RIBs.

From the first work of Tamburella et al. [10], the $1+/ \mathrm{N}+$ technique or charge breeding technique in ECRIS [11] and EBIS (electron beam ion source) $[12,13]$ has been intensively studied and found to be complementary. Considering the continuous mode of operation and the intrinsic resolving power of the CIME cyclotron, an ECRIS based charge breeder was found suitable. Therefore, the $14.5 \mathrm{GHz}$ SPIRA L1 ECR charge breeder (SP1 CB), based on a LPSC Phoenix ECRIS [14], was installed in the low energy beam transport (LEBT) during the SPIRAL1 upgrade. The device accepts the 1+ RIBs from the TISS, boosts its charge state and 
extracts the multicharged ions $(\mathrm{N}+)$ very quickly and efficiently. Some of the advantages of this technique are the high acceptance of injected $1+$ beam current (up to $1 \mu \mathrm{A}), 1+$ beam emittance $(\leq 50 \pi \cdot \mathrm{mm} \cdot \mathrm{mrad})$, reliability and ability to produce continuous and pulsed high charge state ion beams.

The $1+$ beam from TISS are slowly injected into SP1 CB via a grounded tube and captured by ECR plasma ions via Coulomb collisions and ionized to higher charge states by successive electron impact ionization. The injected $1+$ ions are said to be captured when their velocity is roughly equal to the average velocity of the ECR plasma ions. The global charge breeding performance of the ion source depends on injection, capture and extraction efficiencies and greatly influenced by the nature of the elements (noble and condensable species). In the case of condensable species, the injected ions condense on the cold walls of the plasma chamber and thus affecting the charge breeding efficiency. In the case of noble gases, the injected ions have a nonnegligible chance of getting recycled from the plasma chamber walls. This explains the role of $1+$ ions capture in the charge breeding efficiency of condensable species. The global efficiency $\eta_{g}$ and the charge breeding efficiency $\eta_{q}$ of a given charge state can be written as

$$
\begin{gathered}
\eta_{g}=\frac{\sum\left(I_{q} / q\right)}{I_{1+}}, \\
\eta_{q}=\frac{\left(I_{q} / q\right)}{I_{1+}} .
\end{gathered}
$$

where $I_{1+}$ is the injected $1+$ ion beam current and $I_{q}$ is the extracted current of charge state $q$ from charge breeder. Delcroix and Bers [15] studied the slowing down of incident species $a$ due to the interaction with ECR plasma species $b$ via ion-ion collisions and formulated a slowing down coefficient (in $\mathrm{m} / \mathrm{s}^{2}$ ) as

$$
\frac{\left\langle\Delta \nu_{a}, \|\right.}{\Delta t}=-\frac{n_{b}}{2 \pi \epsilon_{0}^{2}}\left[\frac{Z_{a} Z_{b} e^{2}}{m_{a}\left\langle v_{b}\right\rangle}\right]^{2}\left(1+\frac{m_{a}}{m_{b}}\right) R\left(u_{a b}\right) \ln \Lambda,
$$

where $n_{b}, m_{a}, m_{b}, v_{b}$ and $Z$ is the plasma density, mass of incident ions, mass of plasma ions, velocity of the plasma ions and charge states of incident ions $a$ and plasma ions $b$, respectively. The background plasma ions are assumed to follow a Maxwellian velocity distribution $\left\langle v_{b}\right\rangle=\sqrt{\frac{2 k T_{i}}{m_{b}}}$. The parameter $\mathrm{R}\left(u_{a b}\right)$ exhibits a maximum when the velocity of incident ions $a$ is approximately equal to the most probable speed of the background plasma ions $b$. Corresponding to the above said condition $\left(v_{a}=v_{b}\right)$, the optimum injection energy of the $1+$ ions $\left(E_{a}\right)$, for the best slowing down, can be estimated [16] as

$$
E_{a}=\frac{m_{a}}{m_{b}} k T_{i}
$$

where $k$ is the Boltzmann constant and $T_{i}$ is the temperature of the background plasma ions. Based on Eq. (4), a fine tuning for optimization of incident $1+$ ions energy is required to slow down and get trapped inside the charge breeder plasma [17]. At SPIRAL1, the energy of the incident $1+$ ion beam is controlled by applying a voltage difference $(\Delta \mathrm{V})$ between the TISS ( $\left.V_{\text {TISS }}\right)$ and the SP1 CB $\left(V_{\mathrm{CB}}\right)$. If the $\Delta \mathrm{V}$ is too high, the $1+$ ion beam may hit the plasma chamber wall or escape from the plasma without being trapped. If too low, the $1+$ beam is reflected by the plasma potential and the axial magnetic fringe field.

Considering the operating range of the CIME cyclotron, charge breeding is required for condensable elements ranging from $\mathrm{Na}$ to $\mathrm{Y}$. Therefore, several experiments were performed to optimize and study the charge breeding efficiencies of light alkali ions in $\mathrm{He}, \mathrm{O}_{2}$ and $\mathrm{H}_{2}$ plasma [18]. In the same article, High charge breeding efficiencies of potassium were found with hydrogen plasma and low charge breeding efficiencies with wide CSD (charge state distribution) has been observed with the support gas of higher mass. The charge breeding efficiency versus $1+$ beam injection energy curves, called as $\Delta \mathrm{V}$ curves, of different alkali elements ( $\mathrm{Rb}, \mathrm{Na}$, and $\mathrm{K}$ ) exhibited a constant optimum injection energy range $(6.5 \mathrm{~V}-7 \mathrm{~V})$ in different plasma species. According to Eqs. (3) and (4), optimum $1+$ injection energy for the best slowing down depends on the ratio of injected $1+$ ions mass to background plasma ions mass and also on the temperature of the plasma ions. This implies that the optimum injection energy of $1+$ beam should vary with the mass and temperature of the background plasma ions. In order to understand these aspects, a deeper understanding of the physics involved in the $1+$ beam-ECR plasma interaction is necessary.

The article therefore presents the application of the numerical code, called Monte Carlo charge breeding (MCBC), aiming at modelling of incident $1+$ beam thermalization and capture in an ECR charge breeder ion source plasma $[19,20]$. It is a full six dimensional (6D) phase space code that allows to study the $1+$ beam slowing down and capture in ECR plasma. The simulation results not only reproduce the experimental trends, but allows one to study the influence of each plasma parameter on charge breeding performances.

\section{EXPERIMENTAL INVESTIGATION WITH SPIRAL1 ECR CHARGE BREEDER}

Charge breeding efficiency experiments were performed using the experimental setup as shown in Fig. 1. A thermionic ion gun, developed at GANIL for the commissioning of the facility, has been installed at the production cave in the SPIRAL1 to produce the $1+$ beams of alkali 


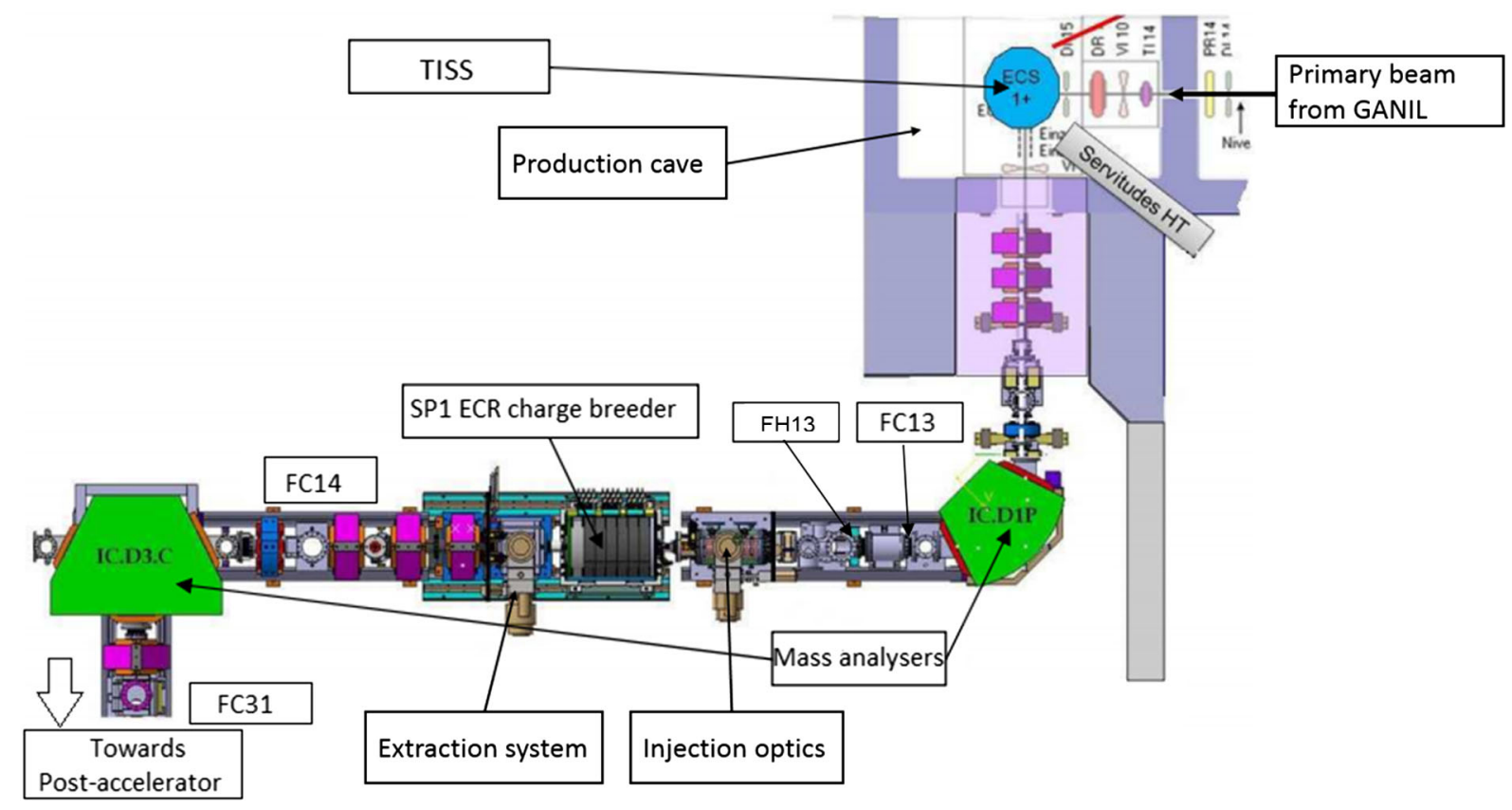

FIG. 1. Schematic layout of the experimental setup at SPIRAL1 facility.

elements. Due to the limited access to the $1+$ source and the SP1 CB, the charge breeding efficiency measurements were only performed with ${ }^{23} \mathrm{Na}^{1+}$ and ${ }^{39} \mathrm{~K}^{1+}$ ion beams at an extraction voltage of $V_{\mathrm{Na}^{1+}}=+(20 \mathrm{kV}+\Delta \mathrm{V})$ and $V_{\mathrm{K}^{1+}}=+(15 \mathrm{kV}+\Delta \mathrm{V})$. Experiments were performed on the following cases: (i) $\mathrm{Na}^{1+}$ beam into He plasma, (ii) $\mathrm{K}^{1+}$ beam into He plasma, and (iii) $\mathrm{K}^{1+}$ beam into $\mathrm{O}_{2}$ plasma. The charge breeding of $\mathrm{Na}^{1+}$ into $\mathrm{O}_{2}$ plasma was avoided due to unfavorable gas mixing conditions.

TABLE I. Charge breeding efficiency results (percentages) obtained from the three experimental cases. The relative uncertainty is about $5 \%$ for the low charge states $(1+$ and $2+)$ and $10 \%$ for the high charge states $(8+$ and $9+)$.

\begin{tabular}{lcccc}
\hline \hline Cases & $1+$ & $2+$ & $8+$ & $9+$ \\
\hline $\mathrm{Na}^{1+}+\mathrm{He}$ & 3.1 & 0.9 & 3.63 & $\ldots$ \\
$\mathrm{K}^{1+}+\mathrm{He}$ & 2.5 & 1.35 & $\ldots$ & 6.53 \\
$\mathrm{~K}^{1+}+\mathrm{O}_{2}$ & 6.4 & 1.28 & $\ldots$ & 1.0 \\
\hline \hline
\end{tabular}

The $1+$ ions from the production cave are selected by a $90^{\circ}$ mass analyzer dipole (D1) and steered through a set of slits (FH13) where the geometrical emittance $(4 \sigma)$ is defined as $50 \pi$.mm.mrad. The $1+$ ions are focused again by injection optics (an electrostatic quadrupole triplet) and injected into SP1 CB with a typical intensity of $500 \mathrm{nA}$. The charge bred ions are extracted through extraction system and the required high charge state ions are selected by the $90^{\circ}$ mass analyzer dipole in the $\mathrm{N}+$ line. Finally, the charge breeding efficiency is calculated using Eq. (2). The $\Delta \mathrm{V}$ parameter is properly optimized to inject the incident $1+$ beam through the axial magnetic fringe field and positive plasma potential of the SP1 CB and achieve maximum charge breeding efficiency. The charge breeding efficiency results and charge breeding parameters for the three cases are presented in the Table I and II.

The experimentally recorded $\Delta \mathrm{V}$ curves of various charge states of $\mathrm{Na}$ and $\mathrm{K}$ in $\mathrm{He}$ and $\mathrm{O}_{2}$ plasma are shown in Fig. 2. It should be noted that the mass analyzer dipole

TABLE II. Typical optimum parameters obtained from the charge breeding efficiency measurements.

\begin{tabular}{lccc}
\hline \hline Parameters & $\mathrm{Na}^{1+}+\mathrm{He}$ & $\mathrm{K}^{1+}+\mathrm{He}$ & $\mathrm{K}^{1+}+\mathrm{O}_{2}$ \\
\hline$V_{\text {TISS }}[\mathrm{V}]$ & 20000 & 15000 & 15000 \\
$V_{\mathrm{CB}}[\mathrm{V}]$ & 20000 & 15000 & 15000 \\
$\Delta \mathrm{V}[\mathrm{V}]$ & 6.5 & 7.5 & 6.5 \\
$\mathrm{RF}$ power [W] & 470 & 207 & 209 \\
Extraction Einzel [kV] & 11.5 & 10.4 & 10.4 \\
B-field (inj/min/ext) [T] & $1.38 / 0.39 / 0.94$ & $1.38 / 0.41 / 0.88$ & $1.38 / 0.40 / 0.88$ \\
Injection triplet [kV] & $\pm(1.15 / 0.7 / 0.25)$ & $\pm(1.3 / 1.7 / 0.35)$ & $\pm(1.3 / 1.7 / 0.35)$ \\
\hline \hline
\end{tabular}




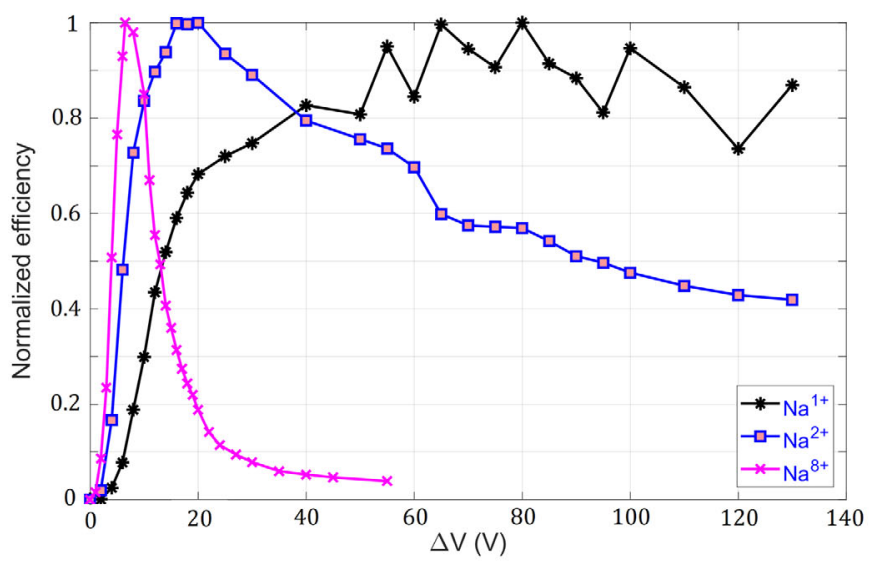

(a)

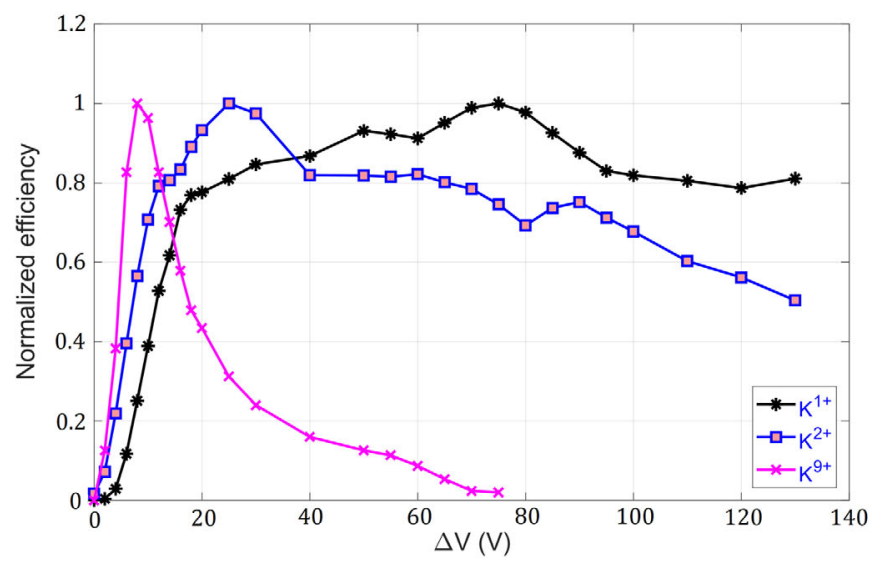

(b)

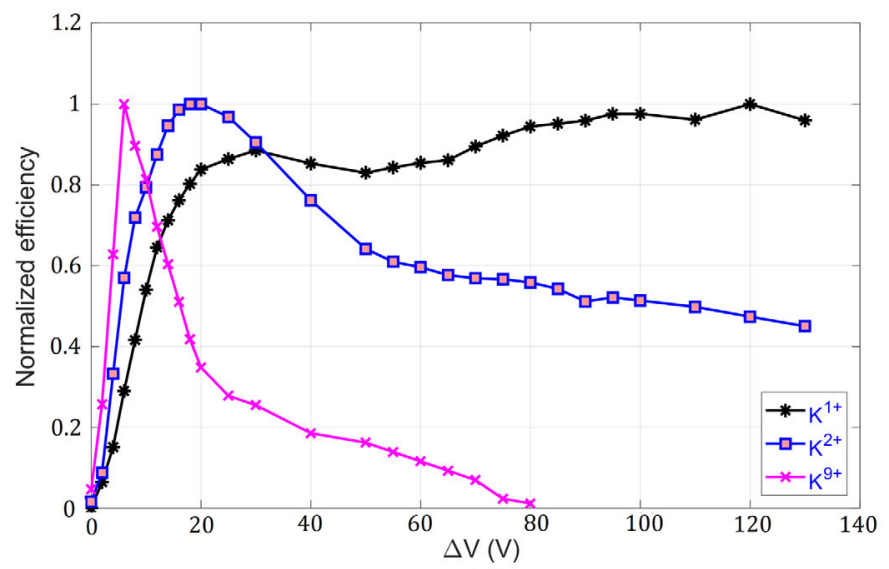

(c)

FIG. 2. Normalized experimental $\Delta \mathrm{V}$ curves of various charge states of (a) ${ }^{23} \mathrm{Na}$ in $\mathrm{He}$ plasma, (b) ${ }^{39} \mathrm{~K}$ in $\mathrm{He}$, and (c) ${ }^{39} \mathrm{~K}$ in $\mathrm{O}_{2}$ plasma.

(D1) in the $1+$ line has been tuned for each $\Delta \mathrm{V}$ in order to maintain the alignment of the $1+$ beam. The efficiency of singly charged ions rapidly increases up to $\Delta \mathrm{V}=40 \mathrm{~V}$ and starts to exhibit periodic variations at higher voltages. At the same time, the trends of $2+$ curves increases up to $\Delta \mathrm{V}=20 \mathrm{~V}$ and decreases gradually at higher $1+$ injection energies. The trends of high charge state ions are completely different, where the efficiency drops after $\Delta \mathrm{V}=30 \mathrm{~V}$. It can be observed that the optimum $\Delta \mathrm{V}$ value for high charge states of $\mathrm{Na}$ and $\mathrm{K}$ is between 6.5 and $7.5 \mathrm{~V}$. Experimental studies have demonstrated that the trends of $1+\Delta \mathrm{V}$ curves represents a fraction of uncaptured incident $1+$ beam propagating through the charge breeder ECR plasma, and the $2+$ are considered to represent in-flight ionization by electron impact and/or $1+$ ions can be captured and ionized to $2+$ [21]. On the other hand, the $\Delta \mathrm{V}$ curves of high charge states $(8+$ and $9+$ ) represents the ion population that has been captured by the background plasma ions and ionized by consecutive electron impacts. The experimental data obtained from the three case studies are used in the numerical simulations to reproduce and compare the trends of experimentally recorded $\Delta \mathrm{V}$ curves.

\section{NUMERICAL MODELING OF $1+$ ION BEAM INTERACTION IN AN ECR PLASMA USING MCBC}

MCBC has been developed by an American based company "FAR-TECH, Inc." in collaboration with ion source group from Argonne National Laboratory (ANL). The code can track the trajectories of injected $1+$ ions under the influence of electric and magnetic fields in an ECR plasma, it models the Coulomb collisions and also the atomic physics processes (electron impact ionization and charge exchange) until the injected $1+$ ions are captured or lost. At the end of each run, the code can give the extracted particle positions, velocities and their energies in a separate output file. In the code, the incident $1+$ ion beam entering the plasma domain are first slowed down and experience multiple interactions with the particles in the background plasma. The beam dynamics between two successive collisions follows the equation of motion which is written as

$m_{a} \frac{d \vec{v}}{d t}=q_{a} \vec{E}(\vec{r})+q_{a} \vec{v} \times \vec{B}(\vec{r})+$ Coulomb collision,

where $m_{a}$ and $q_{a}$ is the mass and charge of the injected test particle $(a)$ propagating with a velocity $\left(v_{a}\right), \vec{r}$ is the 
position vector, $\vec{v}$ is the velocity vector, $\vec{E}=-\nabla \phi$ is the electric field and $\vec{B}$ is the external magnetic field. The formulations given by Tribnikov [22,23] are considered to treat the Coulomb collisions between the injected test particle $a$ propagating with a velocity $\left(v_{a}\right)$ and Maxwellian background plasma particles $(b)$ of mass $m_{b}$ propagating with a velocity $\left(v_{b}\right)$. The slowing down rate $\left(\nu_{s}\right)$, perpendicular diffusion rate $\left(\nu_{\perp}\right)$ and parallel diffusion rate $\left(\nu_{\|}\right)$resulting from the Coulomb collisions are written as

$$
\begin{gathered}
\nu_{s}^{a / b}=\left(1+\frac{m_{a}}{m_{b}}\right) \psi(x) \nu_{0}^{a / b} \\
\nu_{\perp}^{a / b}=2\left[\left(1-\frac{1}{2 x}\right) \psi(x)+\psi^{\prime}(x)\right] \nu_{0}^{a / b} \\
\nu_{\|}^{a / b}=\left[\frac{\psi(x)}{x}\right] \nu_{0}^{a / b}
\end{gathered}
$$

The term $\nu_{0}^{a / b}$ refers to the fundamental Coulomb collision frequency for all the processes. The density of injected $1+$ ions is assumed to be much smaller than that of background plasma ions (i.e., no interaction between injected $1+$ ions). The parameter $\psi(\mathrm{x})$, originated from the work on stellar collisions [24,25], in the Eqs. (6), (7), and (8) is conveniently written in terms of Maxwell integral as

$$
\begin{gathered}
\psi(x) \equiv \frac{2}{\pi} \int_{0}^{x} d t \sqrt{t} e^{-t}, \\
\psi^{\prime} \equiv \frac{d \psi}{d x}=2 \sqrt{\frac{x}{\pi}} e^{-x}, \\
\psi+\psi^{\prime}=\operatorname{erf}(\sqrt{x}),
\end{gathered}
$$

in which $x \equiv x^{a / b}=\frac{m_{b} v_{a}^{2}}{2 k T_{i}}$. Due to the difference in the mass, the beam slowing down due to the Coulomb collisions with plasma electrons is orders of magnitude smaller than the one due to plasma ions. Therefore, the slowing down mechanism due to plasma electrons in MCBC is neglected. The function $\psi(\mathrm{x})$ shows a maximum $(x=1)$ when the test particle velocity is equal to the most probable thermal speed of background plasma ion.

\section{A. Monte Carlo Coulomb collision operator by Boozer}

Equations (6), (7), and (8) provide a complete description of the evolution of incident $1+$ ions suffering Coulomb collisions with the background plasma ions. Applying these equations, Boozer has derived a convenient long range Coulomb collision formula for Monte Carlo simulations of beam slowing and scattering [26,27]. The Boozer formula can be represented in two separate operations: the first operator changes the incident $1+$ ion beam velocity vector without changing its magnitude which is expressed as

$$
\begin{aligned}
v_{\text {new }}= & \left(1-\nu_{\perp}^{a / b} \frac{\tau}{2}\right) v_{\text {old }} \hat{v} \\
& +\sqrt{\left(1-\frac{1}{4} \nu_{\perp}^{a / b} \tau\right) \nu_{\perp}^{a / b} \frac{\tau}{2}}\left( \pm \hat{\varphi} \pm \hat{\theta} v_{\text {old }}\right)
\end{aligned}
$$

where $\hat{v} \equiv v_{\text {old }} /\left|v_{\text {old }}\right|, \hat{\varphi}$ and $\hat{\theta}$ are the orthonormal vectors in velocity space, $\nu_{\perp}$ is the ion-ion collision frequency perpendicular to beam direction. Each \pm refers to the random choice in either direction on each time step $\tau$ with equal probability of each sign. Equation (12) changes the direction of ion velocity while conserving the ion energy. The second operator changes the energy as a consequence of beam slowing down and energy diffusion as

$$
\begin{aligned}
E_{n}= & E_{0}-\frac{2 \nu_{s}^{a / b} \tau}{1+m_{b} / m_{a}}\left[E_{0}-\left(\frac{3}{2}+\frac{d \ln \nu_{s}^{a / b}}{d \ln E_{0}}\right) T_{i}\right] \\
& \pm 2 \sqrt{\frac{T_{i} E_{0} \nu_{s}^{a / b} \tau}{1+m_{b} / m_{a}}}
\end{aligned}
$$

with $E_{0} \equiv \frac{1}{2} \mathrm{~m} v_{b}^{2}$ the ion energy in the previous time step. The first term in Eq. (13) describes the ion beam slowing down, the second term describes the resulting energy after the scattering of the injected particles on Maxwellian background plasma particles and the \pm sign refers to a random choice in either direction on each time step $\tau$ with equal probability of each sign. In addition to the Coulomb collisions, the collisions due to atomic physics process such as electron impact ionization [28] and charge exchange recombination [29] are included in MCBC. The code assumes the atomic collisions as a individually separate and distinct event and also treated in a probabilistic way using a rejection technique.

\section{B. Limitations of $\mathrm{MCBC}$}

There are certain limitations of $\mathrm{MCBC}$ which should be mentioned before discussing the simulation strategy: (i) The ionization processes like double ionization, radiative and dielectric recombination processes are not included in the code due to unavailable cross section data for every element. (ii) The code is inaccessible for the user. Therefore, there is no possibility to make additional modifications. (iii) During the Coulomb collisions, the incident $1+$ beam should collide with every ion charge state of the background plasma. This problem has been simplified in MCBC by letting the $1+$ ions collide with one effective plasma ion having an effective charge $\left\langle Z_{\text {eff }}\right\rangle=\sum_{b} I_{b} q_{b} / \sum_{b} I_{b}$, where $I_{b}$ is the intensity of the plasma species which are in charge state $q_{b}$. (iv) The $1+$ ions are injected into the SP1 CB plasma chamber at an arbitrary time $t=0$, and are transported into the charge breeder for a total time of $500 \mu \mathrm{s}$, which allows us to limit the computational time required by $\mathrm{MCBC}$ for performing 
the simulation. This implies that with $\mathrm{MCBC}$ alone the injected ions do not reach charge equilibrium. We note that in [20] additional packages (namely GEM and IONEX) were used to simulate realistic charge state distributions from the ECR plasma.

As we concentrate here on the physics of $1+$ beam capture, the efficiencies for low charge states are the most relevant ones [16,30]. Consequently, MCBC is sufficient for our purpose. Nevertheless, considering all the limitations above, the efficiencies of low charge state ions $(1+$ and $2+)$ obtained from the MCBC can be different in absolute magnitude regarding the experimental recorded efficiencies. Therefore, the $\Delta \mathrm{V}$ curves obtained from simulation and experiments were normalized to enable a shape comparison. As will be shown in the following, adjusting the simulated shape to the experimental one gives access to a quantitative evaluation of important parameters of the plasma.

\section{Simulation domain and strategy}

Figure 3 shows the physical boundaries of the SP1 CB simulation domain (SP1 CB plasma chamber) extending from the tip of the grounded tube of the SP1 CB $(z=0 \mathrm{~m})$ to the extraction aperture $(z=0.328 \mathrm{~m})$. The minimum-B configuration in the plasma domain is created by a set of three solenoid coils and a permanent magnet hexapole. In order to reproduce the experimental trends, realistic beam starting conditions are required for the simulation which can be obtained by the following approach as shown in the Fig. 4.

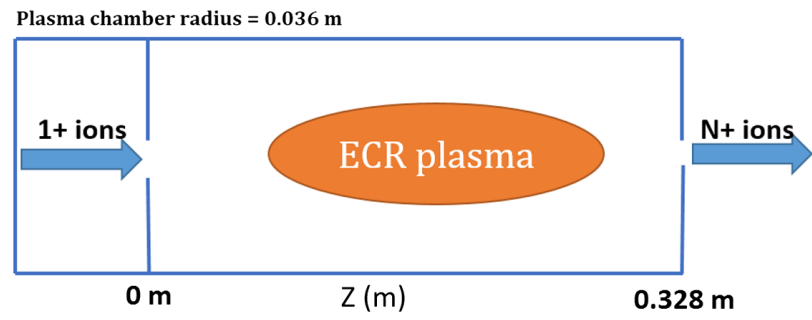

FIG. 3. MCBC simulation domain $(z=0 \mathrm{~m}$ to $0.328 \mathrm{~m})$ with respect to the SP1 CB plasma chamber dimensions.
SIMION [31], a ion beam transport simulation software, is used to initiate the $1+$ ions from the slits position (FH13) and to transport these up to the entrance of SP1 CB plasma chamber (including the magnetic fringe field). The starting conditions (particle positions and velocities) at the entrance of the plasma chamber can be recorded from SIMION and given as an input to MCBC through a separate data file. At the end of the simulation, MCBC records the properties of ions arriving at the plasma electrode in a separate output file. This output file is given as an input to SIMION to simulate the extracted ions from the plasma electrode position of the SP1 CB. Finally, the efficiency is calculated at the exit of SP1 CB extraction system.

Among the three experimental cases, we present here only the simulation of the first case (injection of $\mathrm{Na}^{1+}$ beam into He plasma). The $\Delta \mathrm{V}$ curves from this case are reproduced by varying each plasma parameter $\left(n_{e}, \mathrm{k} T_{i}\right.$, plasma potential, potential dip and $T_{e}$ ) independently. In this way, the plasma parameters can be estimated and useful information like the proportion of 1+ ions captured in ECR plasma and proportion of $1+$ ions lost in the simulation domain can be obtained. As shown later, the estimated plasma parameters allows one to identify the important mechanisms in the $1+$ ion beam capture process and also reveal reasons for the difference in charge breeding efficiencies of different alkali species $(\mathrm{Na}$ and $\mathrm{K}$ ) in different background plasmas.

To extract the set of plasma parameters from the reproduction of $\Delta \mathrm{V}$ curves, a simplified ECR plasma model has been implemented assuming different plasma parameters into two zones: $\mathrm{B} \leq B_{\mathrm{ECR}}$ with $n_{e}$ (in plasmoid) and $\mathrm{B} \geq B_{\mathrm{ECR}}$ with $n_{e} * 10^{-1}$ (halo). This plasma model scheme has been proposed in a few articles [32,33] and implemented in previous approaches adopted in [34,35]. Table III shows the He plasma parameters (reference parameters) acquired from previous approaches $[30,34,35]$. The neutral density in the three experimental cases were calculated from the SP1 CB operating pressures. It is important to mention that the perturbation caused by the $1+$ beam injection on the SP1 CB plasma is relatively small. The effective charge $\left\langle Z_{\text {eff }}\right\rangle$ of $\mathrm{He}$ and $\mathrm{O}_{2}$ plasma has been calculated (without taking impurities into the account)

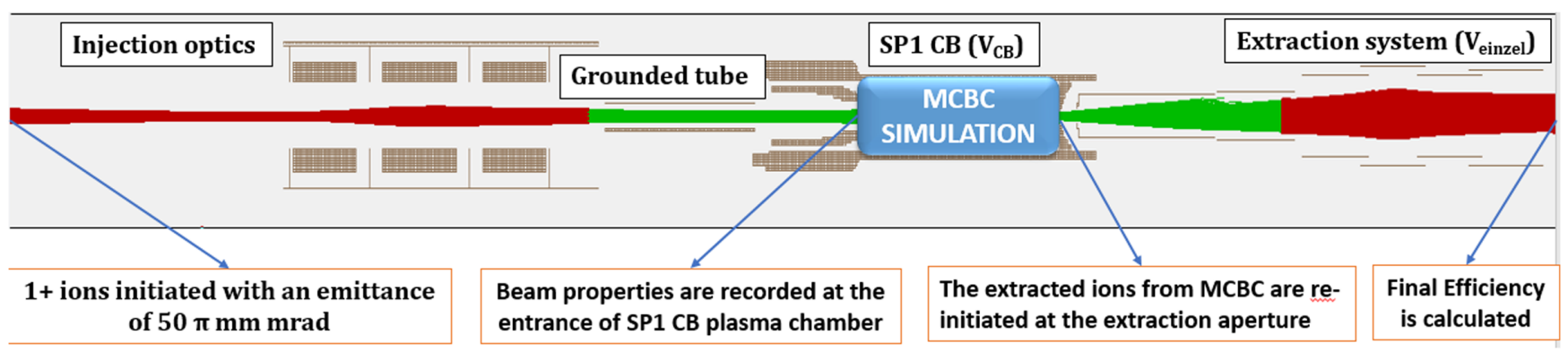

FIG. 4. The simulation approach implemented to perform MCBC simulations. 
TABLE III. Initial He plasma parameters (reference parameters).

\begin{tabular}{lc}
\hline \hline Plasma parameters & Values \\
\hline Number of $\mathrm{Na}^{1+}$ ions & 50000 \\
Support gas & ${ }^{4} \mathrm{He}$ \\
$\mathrm{k} T_{i}[\mathrm{eV}]$ & 0.5 \\
$T_{e}[\mathrm{keV}]$ & 1 \\
$n_{e}\left[\mathrm{~cm}^{-3}\right]$ & $4 \times 10^{11}$ \\
Neutral density $\left[\mathrm{m}^{-3}\right]$ & $3.21 \times 10^{14}$ \\
\hline \hline
\end{tabular}

TABLE IV. Comparison of plasma effective charge $\left\langle Z_{\text {eff }}\right\rangle$ with and without $1+$ injection.

\begin{tabular}{lccc}
\hline \hline & $\mathrm{Na}^{1+}+\mathrm{He}$ & $\mathrm{K}^{1+}+\mathrm{He}$ & $\mathrm{K}^{1+}+\mathrm{O}_{2}$ \\
\hline With 1+ injection & 1.45 & 1.29 & 4.02 \\
Without $1+$ injection & 1.58 & 1.38 & 4.15 \\
\hline \hline
\end{tabular}

from the mass spectrum obtained from the three experimental case studies and presented in the Table IV.

At the same time, the ion density can be calculated as $n_{i}=n_{e} /\left\langle Z_{\text {eff }}\right\rangle$. In addition to the background plasma parameters, the deceleration of the incident $1+$ beam should be taken into account. To this intent, a 3D electric field has been acquired from SIMION geometry as shown in Fig. 5.

The contour of the plasma potential is considered to have a similar geometry as the one of the charge breeder plasma chamber wall. The distance between the chamber wall and sheath is considered as $1 \mathrm{~mm}$. To take into account the deceleration of $1+$ ions due to the positive plasma potential, a plasma potential $\left(V_{P}\right)$ is considered on the top of the charge breeder potential $\left(V_{c b}\right)$. The ECR zone, approximated as cylindrical symmetric ellipsoid surface, formed in minimum-B structures created by the axial and radial magnetic field as shown in Fig. 5. As described in previous articles, the confinement of highly charged ions is improved by the presence of a potential dip in the ECR

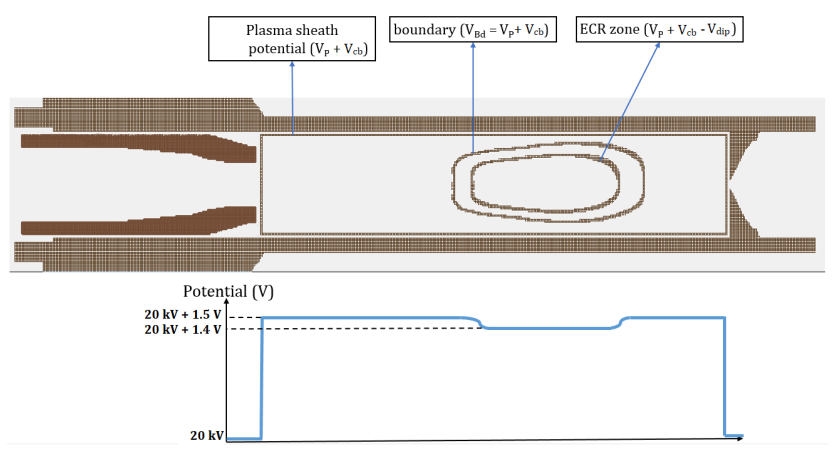

FIG. 5. The modified SP1 CB SIMON geometry with ECR plasma potential features (top figure). The assumed plasma potential distribution for the charge breeding simulations (bottom figure). surface [36]. It has been suggested that a negative charged cloud is formed due to the collisionless hot electron component adiabatically confined by the magnetic field in the ECR plasma. To approximate a potential depression created by the hot electrons in the ECR zone, a potential $\operatorname{dip} V_{\text {dip }}$ value of $0.1 \mathrm{~V}$ in the plasma model, which is considered to be always less than the plasma sheath potential $\left(V_{\mathrm{ECR}}=V_{P}+V_{c b}-V_{\mathrm{dip}}\right)$. Applying the potentials to the electrodes, SIMION generates the three components of the electric field (Ex, Ey, Ez). This scheme allows $1+$ ions into the plasma boundary with an energy $E_{\text {inj }}^{\text {sim }}=e\left(\Delta \mathrm{V}-V_{P}\right)$. The $1+$ ions, reaching the ECR zone, are first accelerated and then decelerated inside the zone. In this way the $1+$ ions reside inside the ECR volume. Ions, residing outside the ECR volume, are accelerated toward the plasma electrode or the chamber wall. Finally, the $\Delta \mathrm{V}$ plot from the simulation can be acquired by varying $\Delta \mathrm{V}$ from $0 \mathrm{eV}$ to $130 \mathrm{eV}$. In this simple assumptions the plasma is therefore assumed as a fixed equipotential surface neglecting the potential drop near the device boundary (presheath effect) and the plasma particles dynamics in the electromagnetic fields.

\section{Simulation of $1+$ beam capture}

In the first set of simulation, $50000 \mathrm{Na}^{1+}$ ions were initiated from beam slits position with a geometrical emittance of $50 \pi \mathrm{mm}$.mrad. They were transported through the injection optics and injected into SP1 CB via grounded tube. $100 \%$ transmission has been recorded up to the entrance of SP1 CB. The energy spread of the thermionic gun used in the experiments is $\approx 2 \mathrm{eV}$ (measured by retarding field analyzer device) which is very small and therefore, neglected in the simulations. With the resulting beam properties at the entrance of SP1 CB plasma chamber, the $1+$ ions are re-initiated in MCBC. The simulation integration time has been set to $500 \mu$ s which corresponds to the typical charge breeding time of $1+$ and $2+$ ions measured with and without plasma. The simulation was performed in $5 \times 10^{5}$ time steps with a time step fraction of $0.001 \mathrm{~ms}$. For every injected beam energy $(0 \mathrm{eV}$ to $130 \mathrm{eV}$ ), the percentage of particle that are lost and captured ions were calculated. MCBC simulations were performed in two steps: In the first step, each plasma parameter was varied independently and the $\Delta \mathrm{V}$ curves obtained from each parameter sweep were compared with the $\mathrm{Na}^{1+}$ experimental curve. The $\chi^{2}$ test [37] has been applied to investigate how well the simulation data fits with the experimental results. The plasma parameter which has a minimum $\chi^{2}$ value was chosen as an optimum value of the corresponding parameter. In a second step, the optimum value of each plasma parameters from the first step were varied until the simulated $\Delta \mathrm{V}$ curve follows the trends of experimental $\Delta \mathrm{V}$ curve. 


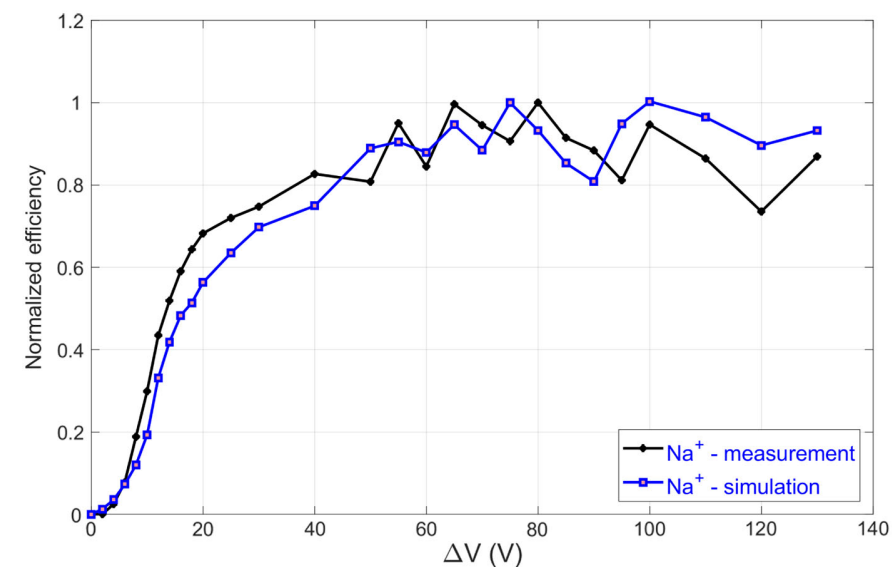

(a)

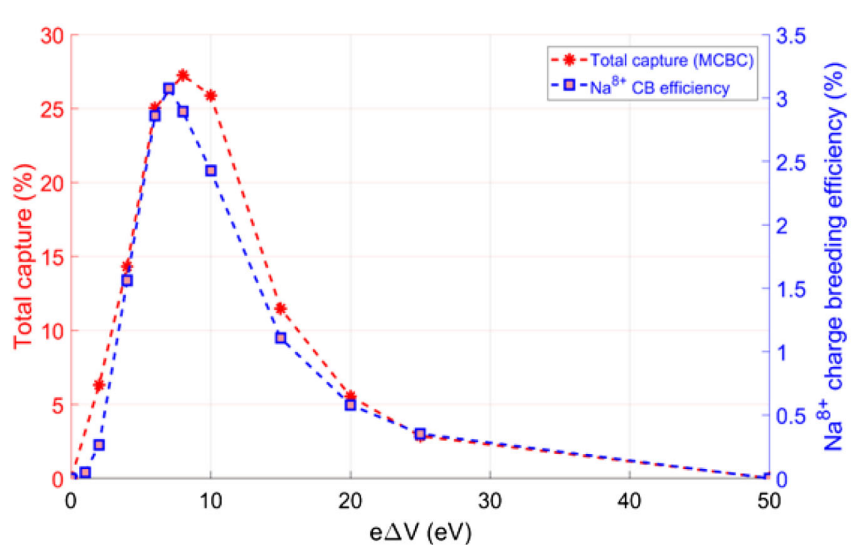

(b)

FIG. 6. (a) Comparison of simulated and experimental $\mathrm{Na}^{1+} \Delta \mathrm{V}$ curves. (b) Comparison between simulated $1+$ ion capture efficiency and experimental $\mathrm{Na}^{8+} \Delta \mathrm{V}$ curve.

The simulations were initiated with the set of reference parameters presented in Table III. Several simulations were performed with rough values of plasma potential and the potential dip. Finally, the plasma potential and potential dip was adjusted to $1.5 \mathrm{~V}$ and $0.5 \mathrm{~V}$. Those parameters were observed to reproduce qualitatively the shape of the experimental $\Delta \mathrm{V}$ curve, as shown in Fig. 6. The shape of the curve is very similar to the experimental ones and exhibits the experimentally observed periodic variations after $40 \mathrm{eV}$. It can be observed from the Fig. 6 that the simulated curve, corresponds to $1+$ ions captured after $500 \mu \mathrm{s}$, peaks at $\mathrm{e} \Delta \mathrm{V}=8 \mathrm{eV}$ and follows the trends of experimental $\mathrm{Na}^{8+} \Delta \mathrm{V}$ curve. Following these observations, it can be said that the $1+$ ions capture plays a dominant role in the charge breeding process. The value of $\mathrm{e} \Delta \mathrm{V}$ found in the simulation is close to the optimum $1+$ injection energy found during the experiments (see Table II).

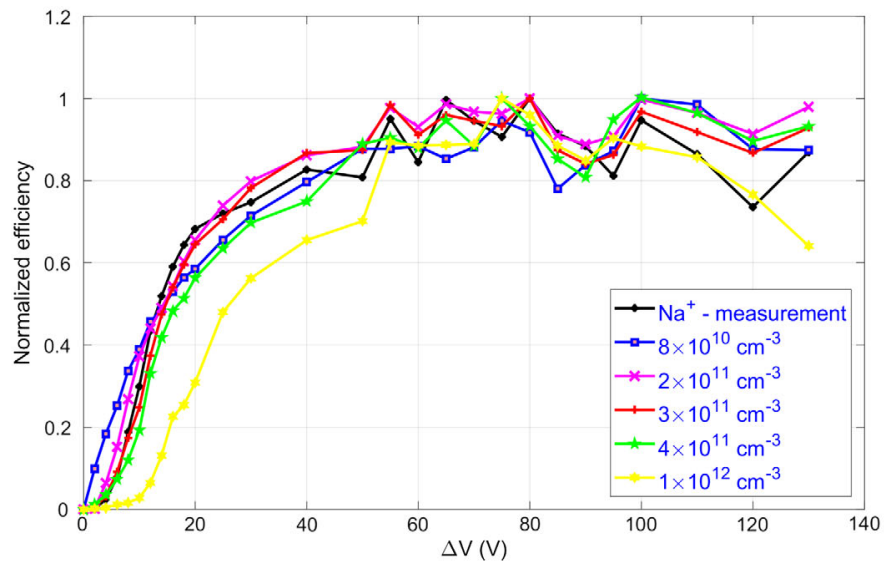

(a)
To reproduce the $\Delta \mathrm{V}$ curve of low charge state ions $(1+$ and $2+)$ and understand the influence of plasma parameters in the capture region $(<20 \mathrm{eV})$, each plasma parameter was then varied independently. In the first sweep, $n_{e}$ was varied from $1 \times 10^{12} \mathrm{~cm}^{-3}$ (near to cutoff density $\approx 2.63 \times 10^{12} \mathrm{~cm}^{-3}$ for $14.5 \mathrm{GHz}$ ) down to $8 \times 10^{10} \mathrm{~cm}^{-3}$. $n_{i}$ was calculated for each value of $n_{e} . \Delta \mathrm{V}$ curves obtained from this sweep are compared with the experimental ones and presented in the figure 7. At higher electron densities $\left(1 \times 10^{12} \mathrm{~cm}^{-3}\right)$, the total capture increases but the shape of the $\Delta \mathrm{V}$ curve is not similar to the experimental ones. At lower densities, the trends of $1+$ curve can be reproduced but with low captured fractions. The $\chi^{2}$ test has been applied to the curves and the minimum $\chi^{2}$ value was found when $n_{e}$ was set to $3 \times 10^{11} \mathrm{~cm}^{-3}$ with an error bar of $\pm 1 \times 10^{11} \mathrm{~cm}^{-3}$. It can be observed that the simulation matches the shape of the curve at the lower injection

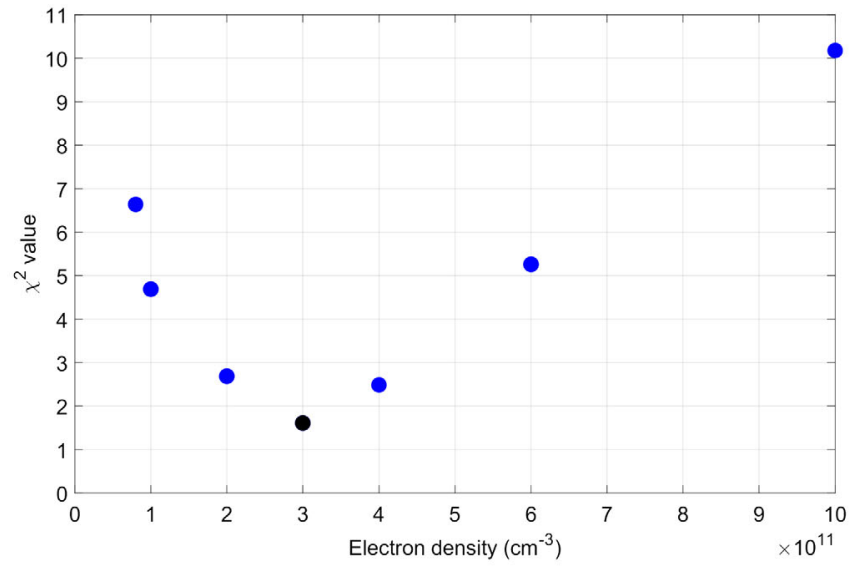

(b)

FIG. 7. (a) Comparison of simulated and experimental $\mathrm{Na}^{1+} \Delta \mathrm{V}$ curves acquired from $n_{e}$ sweep. (b) $\chi^{2}$ plot calculated in the first sweep. The minimum $\chi^{2}$ value (optimum value of $n_{e}$ ) is marked in black. 


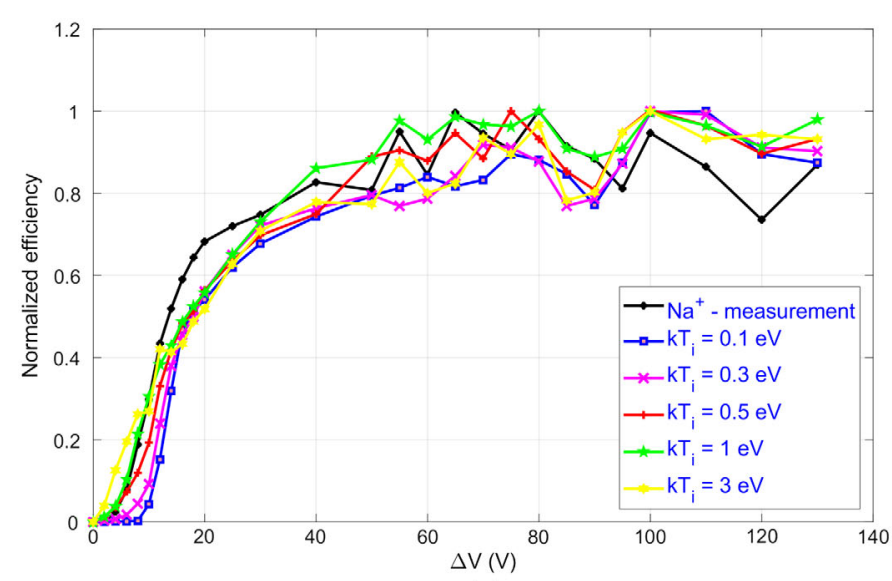

(a)

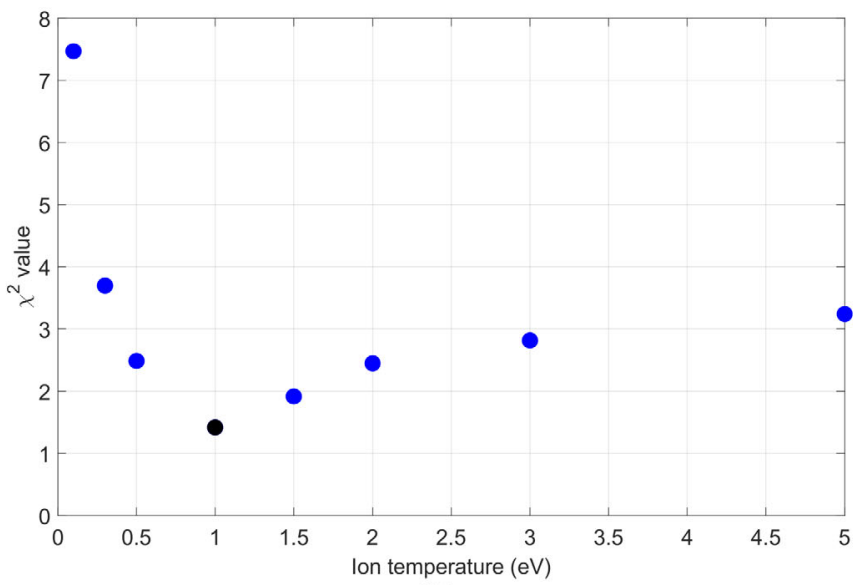

(b)

FIG. 8. (a) Comparison of simulated and experimental $\mathrm{Na}^{1+} \Delta \mathrm{V}$ curves acquired from $\mathrm{k} T_{i}$ sweep. (b) $\chi^{2}$ plot calculated in the first sweep. The minimum $\chi^{2}$ value (optimum value of $\mathrm{k} T_{i}$ ) is marked in black.

energies and the trend at higher injection energies is also reproduced but with a small discrepancy.

After the $n_{e}$ sweep, the influence of $\mathrm{k} T_{i}$ was investigated by varying from $0.1 \mathrm{eV}$ to $3 \mathrm{eV}$. Due to the strong dependence of ion temperature, the ion-ion collisions are more frequent than the other collision frequencies in ECR plasma [38]. As the ion temperature increases, the collisions becomes less frequent $\left(\nu_{i i} \propto k T_{i}^{-3 / 2}\right)$. Therefore, a great influence of this parameter on the $1+$ capture can be expected. For each value of $\mathrm{k} T_{i}$, the $\Delta \mathrm{V}$ curve has been obtained and compared with the experimental curve and presented in the Fig. 8. As expected, the parameter has shown a great influence in the capture region. When the temperature was set to $0.1 \mathrm{eV}$, the $1+$ ions are captured in the plasma and not extracted at the end of the simulation even at the injection energy of $10 \mathrm{eV}$. Moreover, the shape of the curve was observed to be far from experimental one. As the ion temperature increases up to $1 \mathrm{eV}$ range, the

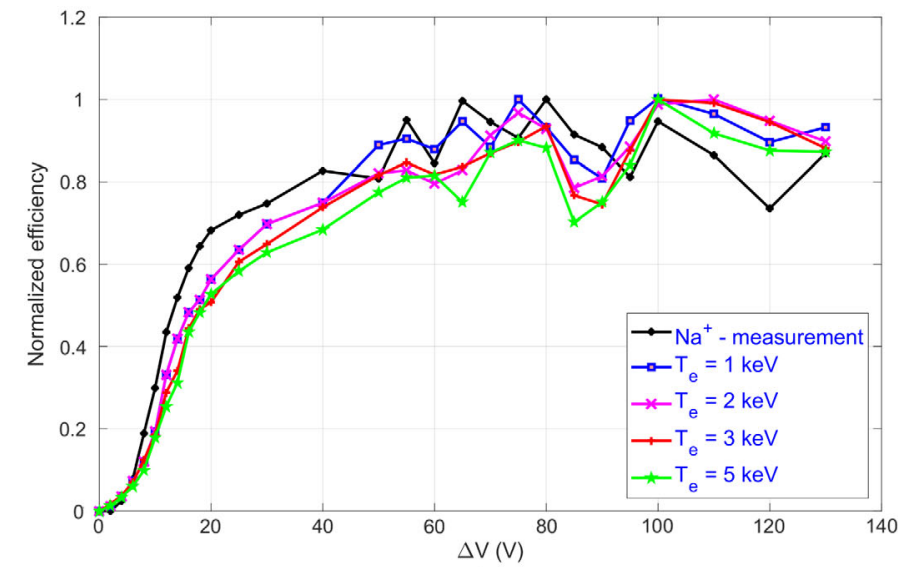

(a) simulated curves at lower injection energies starts moving toward the experimental curve. In the higher values of $\mathrm{k} T_{i}$ ( 1.5 to $5 \mathrm{eV}$ ), the confinement is very poor due to low collision frequencies with the plasma ions. A small variation in $\mathrm{k} T_{i}$ exhibits different trends at lower and higher injection energies. The $\chi^{2}$ value has been calculated for each case and the minimum value was found when the $\mathrm{k} T_{i}$ was set to $1 \mathrm{eV}$ with an error bar of $\pm 0.5 \mathrm{eV}$. Following the variation of $\mathrm{k} T_{i}, T_{e}$ was varied by assuming warm electrons component in the core $(1 \mathrm{keV}, 2 \mathrm{keV}, 3 \mathrm{keV}$ and $5 \mathrm{keV})$. The results obtained with these temperatures are compared with the experimental results and presented in Fig. 9. While increasing $T_{e}(>1 \mathrm{keV})$, simulations revealed no significant change in the shape of simulated curves at low injection energies and different trends at higher injection energies. The minimum $\chi^{2}$ was found when $T_{e}$ set to $1 \mathrm{keV}$ as an optimum with an error bar of $\pm 0.5 \mathrm{keV}$. The negligible influence on the $\Delta \mathrm{V}$ curves

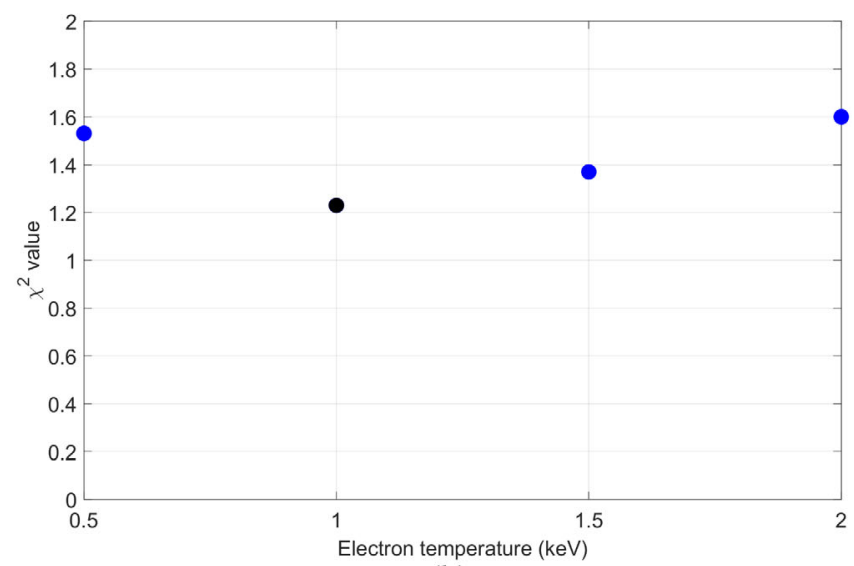

(b)

FIG. 9. (a) Comparison of simulated and experimental $\mathrm{Na}^{1+} \Delta \mathrm{V}$ curves acquired from $T_{e}$ sweep. (b) $\chi^{2}$ plot calculated in the first sweep. The minimum $\chi^{2}$ value (optimum value of $T_{e}$ ) is marked in black. 
TABLE V. Calculated ionization times [39] inside the plasmoid for Sodium charge states from 0 to $3+$ considering different electron temperatures.

\begin{tabular}{lcccc}
\hline \hline & \multicolumn{4}{c}{$\tau_{\text {ionization }}(\mu \mathrm{s})$} \\
\cline { 2 - 5 } Ionization & $1 \mathrm{keV}(\mu \mathrm{s})$ & $2 \mathrm{keV}$ & $3 \mathrm{keV}$ & $5 \mathrm{keV}$ \\
\hline $0 \rightarrow 1+$ & 37.1 & 46.4 & 53.2 & 63.6 \\
$1+\rightarrow 2+$ & 116 & 133 & 147 & 169 \\
$2+\rightarrow 3+$ & 207 & 232 & 253 & 288 \\
$3+\rightarrow 4+$ & 362 & 394 & 425 & 477 \\
\hline \hline
\end{tabular}

TABLE VI. Optimum plasma parameters obtained in second step.

\begin{tabular}{lc}
\hline \hline Parameters & $\mathrm{Na}^{1+}+\mathrm{He}$ \\
\hline $\mathrm{e} \Delta \mathrm{V}[\mathrm{eV}]$ & 7.5 \\
$V_{P} / V_{\mathrm{dip}}[\mathrm{V}]$ & $1.5 / 0.5$ \\
$n_{e}\left[\mathrm{~cm}^{-3}\right]$ & $3.3-3.7 \times 10^{11}$ \\
$\mathrm{k} T_{i}[\mathrm{eV}]$ & $0.9-1.1$ \\
$T_{e}[\mathrm{keV}]$ & $1-2$ \\
$\left\langle Z_{\mathrm{eff}}\right\rangle$ & 1.5 \\
Neutral density $\left(\mathrm{m}^{-3}\right)$ & $3.21 \times 10^{14}$ \\
\hline \hline
\end{tabular}

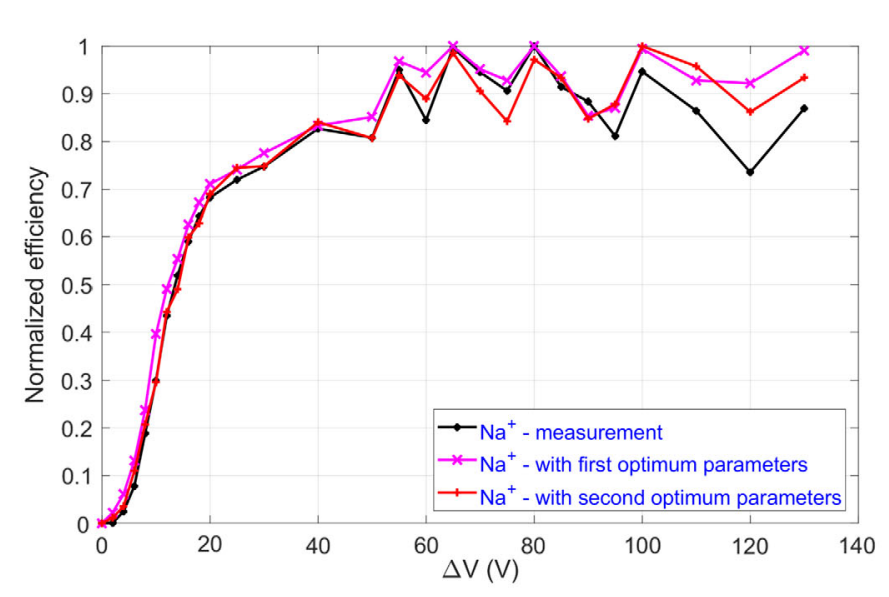

(a) observed during the variation of this parameter is due to low ionization potentials of the corresponding $1+$ ions. The different temperatures used in the simulation roughly lead to similar ionization times as shown in the table V. The ionization times for the first four charge states are shorter than the time span of the simulation $(500 \mu \mathrm{s})$.

The optimum value obtained for each plasma parameter, thanks to the sweeps, was then further optimized to find the correct parameters that can reproduce the experimental $\mathrm{Na}^{1+} \Delta \mathrm{V}$ curve. The optimum plasma parameters found in this second step are shown in Table VI. substituting the mean value from optimum values, a very good agreement between the simulated and experimental $\mathrm{Na}^{1+} \Delta \mathrm{V}$ curve can be seen in Fig. 10. In the same figure, a fraction of $1+$ ions captured using the initial plasma parameters (reference parameters) and final plasma parameters (from second sweep) is presented. The optimum $\mathrm{e} \Delta \mathrm{V}$ is $7.5 \mathrm{eV}$ which is a bit higher than the optimum $\Delta \mathrm{V}$ found during the experiment $(6.5 \mathrm{~V})$. It can be seen that the total capture is reduced by a factor of 1.7 when compared with results obtained using the reference parameters. The difference between the reference parameters and the final

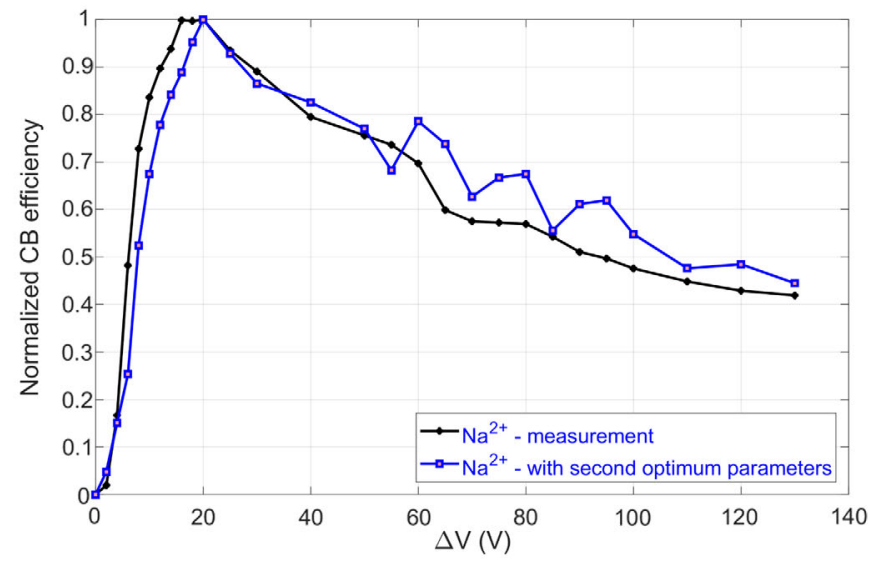

(b)

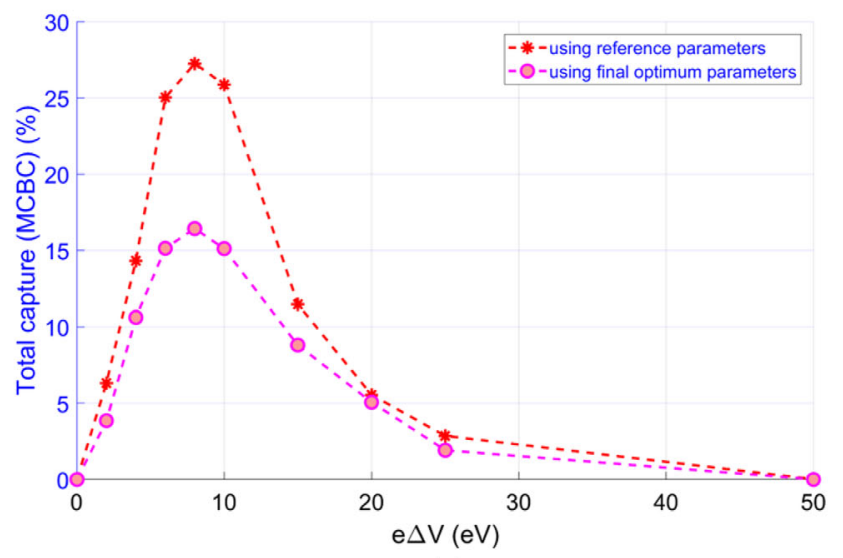

(c)

FIG. 10. (a) Comparison of experimental and simulated $\mathrm{Na}^{+} \Delta \mathrm{V}$ curves acquired using final optimum plasma parameters. (b) Comparison of experimental and simulated $\mathrm{Na}^{2+} \Delta \mathrm{V}$ curves acquired using final optimum plasma parameters. (c) Proportion of $1+$ ions captured as a function of $\mathrm{e} \Delta \mathrm{V}$ using the initial and final plasma parameters. 
TABLE VII. Final optimum parameters used in the simulations to reproduce the $\mathrm{K}^{1+}$ and $\mathrm{K}^{2+} \Delta \mathrm{V}$ curves.

\begin{tabular}{lcc}
\hline \hline Parameters & $\mathrm{K}^{1+}+\mathrm{He}$ & $\mathrm{K}^{1+}+\mathrm{O}_{2}$ \\
\hline $\mathrm{e} \Delta \mathrm{V}[\mathrm{eV}]$ & 8.5 & 8.5 \\
$V_{P} / V_{\mathrm{dip}}[\mathrm{V}]$ & $1 / 0.5$ & $1 / 0.7$ \\
$n_{e}\left[\mathrm{~cm}^{-3}\right]$ & $1.5-2.5 \times 10^{11}$ & $1.0-2.0 \times 10^{11}$ \\
$\mathrm{k} T_{i}[\mathrm{eV}]$ & $0.7-0.8$ & $1.5-2.5$ \\
$T_{e}[\mathrm{keV}]$ & $1-2$ & $2-3$ \\
$\left\langle Z_{\text {eff }}\right\rangle$ & 1.38 & 4.15 \\
Neutral density $\left[\mathrm{m}^{-3}\right]$ & $3.21 \times 10^{14}$ & $2.59 \times 10^{14}$ \\
\hline \hline
\end{tabular}

optimum parameters is the $n_{e}$ (from $4 \times 10^{11} \mathrm{~cm}^{-3}$ to $3.5 \times 10^{11} \mathrm{~cm}^{-3}$ ) and the $\mathrm{k} T_{i}$ (from $0.5 \mathrm{eV}$ to $1 \mathrm{eV}$ ), which exhibited a great influence on total $1+$ capture.

Using this set of parameters, the experimental trends of $\mathrm{Na}^{2+} \Delta \mathrm{V}$ curve has been reproduced by increasing the simulation integration time from $500 \mu \mathrm{s}$ to $700 \mu \mathrm{s}$. This

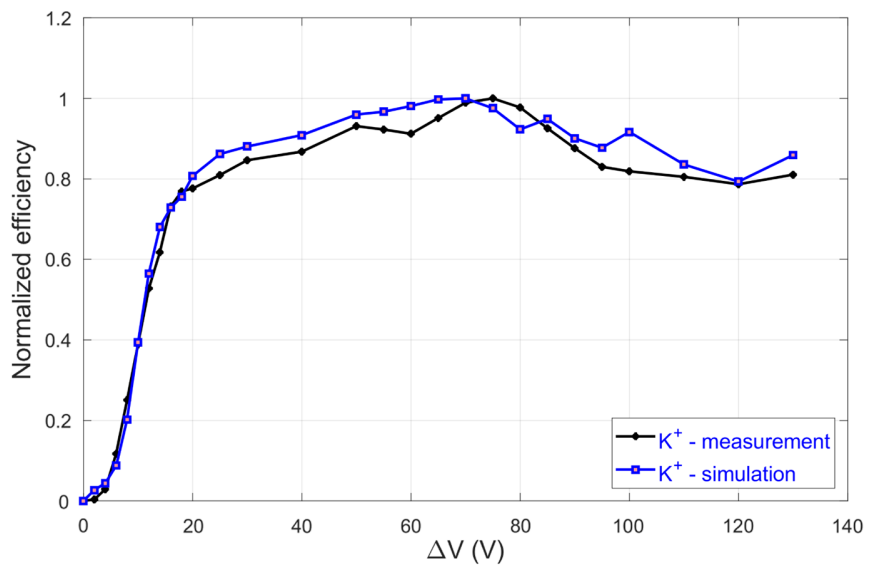

(a) modification has been done considering that the charge breeding time of $2+$ is of the order of $700 \mu$ s. This value has been estimated using the charge breeding time of $\mathrm{K}^{2+}$ in He plasma indicated at the optimum SP1 CB operating conditions. Thus, latter was found to be around $600 \mu \mathrm{s} \pm$ $16 \%$ [40]. The $2+$ charge breeding time for the cases $\left(\mathrm{Na}^{1+}+\mathrm{He}\right)$ and $\left(\mathrm{K}^{1+}+\mathrm{O}_{2}\right)$ has been assumed to be in the similar range as $\mathrm{K}^{2+}$ in He plasma. Figure 10 shows the comparison of $\mathrm{Na}^{2+} \Delta \mathrm{V}$ curve obtained from the simulation with the experimental $\mathrm{Na}^{2+} \Delta \mathrm{V}$ curve. At injection energies lower than $40 \mathrm{eV}$, the comparison shows a better agreement with the experimental curve. The periodic variations in the higher injection energies exhibits deviations from the experimental curve.

\section{E. $\mathrm{K}^{1+}$ beam capture in $\mathrm{He}$ and $\mathrm{O}_{2}$ plasma}

Following the numerical analysis of $\mathrm{Na}^{1+}$ interaction in He plasma, numerical simulations of the $\mathrm{K}^{1+}$ capture in $\mathrm{He}$

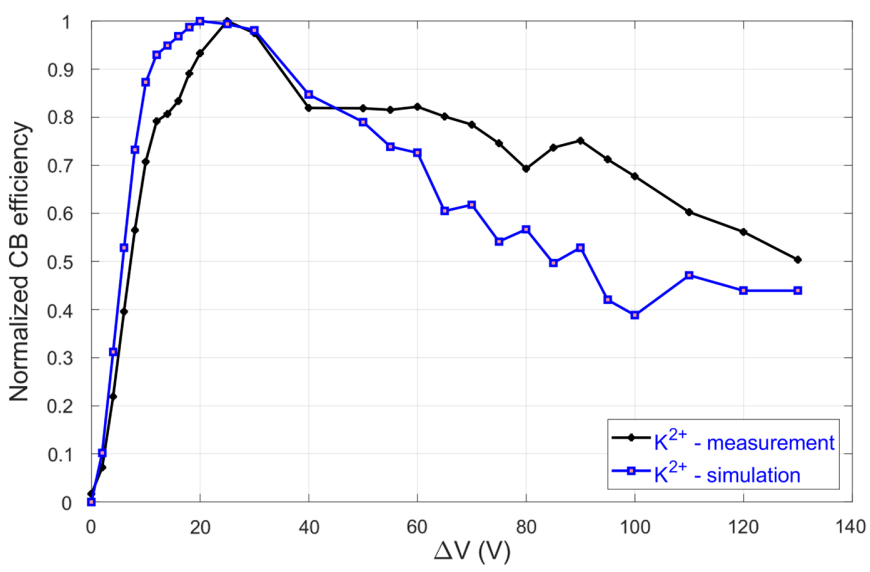

(b)

FIG. 11. Comparison of experimental and simulated (a) $K^{1+}$ and (b) $\mathrm{K}^{2+} \Delta \mathrm{V}$ curves obtained from He plasma parameters. $n_{e}$ of $2 \times 10^{11} \mathrm{~cm}^{-3}$ (for $207 \mathrm{~W}$ of $\mathrm{rf}$ power) and $\mathrm{k} T_{i}$ of $0.75 \mathrm{eV}$ were used in the simulation.

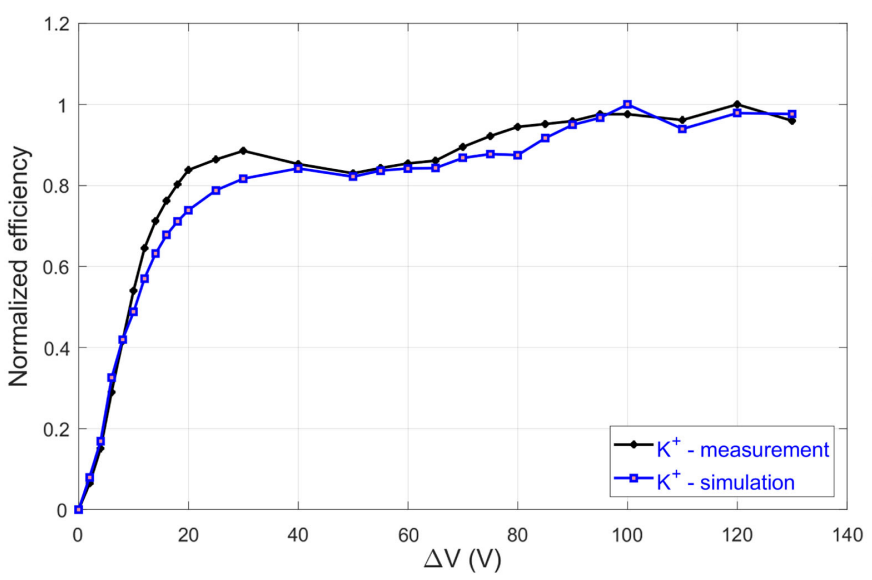

(a)

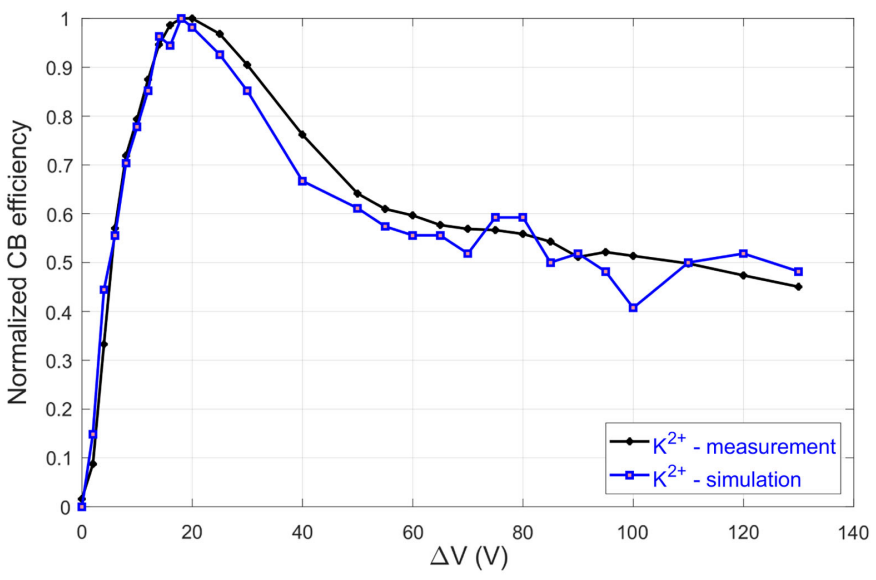

(b)

FIG. 12. Comparison of simulated and experimental (a) $\mathrm{K}^{1+}$ and (b) $\mathrm{K}^{2+} \Delta \mathrm{V}$ curves obtained from $\mathrm{O}_{2}$ plasma parameters. $n_{e}$ of $1.5 \times 10^{11} \mathrm{~cm}^{-3}$ (for $209 \mathrm{~W}$ of $\mathrm{rf}$ power) and $\mathrm{k} T_{i}$ of $2 \mathrm{eV}$ were used in the simulation. 


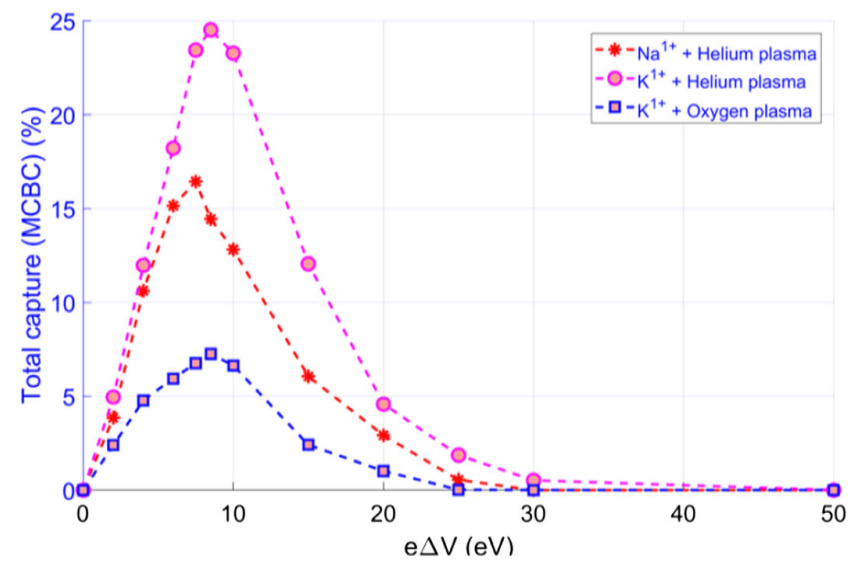

FIG. 13. The estimation of total $1+$ ion capture (in three cases) obtained from MCBC simulations.

and $\mathrm{O}_{2}$ plasma were performed by applying the simulation strategy discussed in the previous section. The input parameters including the magnetic field configuration and potentials for the two experimental cases were taken from Table II. In the two cases, the simulation integration time was increased to $700 \mu$ s in order to reproduce the $1+$ and also $2+\Delta \mathrm{V}$ curves. The plasma parameter variations were performed in two steps as in the case of $\mathrm{Na}^{1+}$ and the $\chi^{2}$ values were calculated during each parameter sweep. The final optimum parameters are shown in Table VII.

Figures 11 and 12 shows the comparison between normalized simulated and experimental $\mathrm{K}^{1+}$ and $\mathrm{K}^{2+}$ $\Delta \mathrm{V}$ curves. The simulated $1+$ and $2+\Delta \mathrm{V}$ trends are in good agreement with the experimentally observed trends at low injection energies $(<40 \mathrm{eV})$ and show some discrepancies in the higher injection energies (mainly in the case of $\mathrm{K}^{2+}$ in He plasma). Figure 13 shows the comparison of the total capture obtained from the three cases. The maximum capture efficiencies of $\mathrm{Na}^{1+}$ and $\mathrm{K}^{1+}$ in $\mathrm{He}$ plasma at different microwave powers $(470 \mathrm{~W}$ and $200 \mathrm{~W})$ are approximately $16 \%$ and $25 \%$, respectively, while the corresponding value for $\mathrm{K}^{1+}$ ions in an $\mathrm{O}_{2}$ plasma with $207 \mathrm{~W}$ rf power is approximately $7 \%$. The optimum e $\Delta \mathrm{V}$ in the three cases is between 7.5 and $8.5 \mathrm{eV}$ which is close to the experimental ones.

\section{DISCUSSION}

Numerical simulations of three cases permitted to reproduce the experimental trends and to extract a set of plasma parameters. In the three simulation cases, the periodic variations exhibited in $1+\Delta \mathrm{V}$ curves at injection energies higher than $40 \mathrm{eV}$ are in good agreement with the variations seen in the experimental curves (especially with $\mathrm{Na}^{1+}$ ). It has to be remembered that the trends of $2+\Delta \mathrm{V}$ curves are reproduced using the set of optimum plasma parameters found in the successful reproduction of experimental $1+\Delta \mathrm{V}$ curves. The only parameter that was modified is the time span of the simulation (changed from $500 \mu \mathrm{s}$ to $700 \mu \mathrm{s}$ ). The periodic variations in $2+$ simulated $\Delta \mathrm{V}$ curves at higher injection energies $(>50 \mathrm{eV})$ deviate somewhat from the experimental ones (especially in the case of $\mathrm{K}^{2+}$ in He plasma). These deviations were also observed in simulated $1+\Delta \mathrm{V}$ curves but the discrepancy was found to be very small when compared with simulated $2+\Delta \mathrm{V}$ curves. This may be due to the simplified plasma model implemented in the simulations.

The plasma density and temperatures profiles presented in the model are very simple compared to the plasma profiles reported in [41]. Nevertheless, it is interesting to analyze more in detail the influence of each input parameter and the plasma parameter on the final capture efficiencies given by MCBC at optimum injection energies.

Simulations revealed different $\mathrm{He}$ and $\mathrm{O}_{2}$ plasma densities at different microwave power. The obtained plasma densities are an order of magnitude lower in comparison to the critical density of $n_{e, \text { critical }} \approx 2.6 \times 10^{12} \mathrm{~cm}^{-3}$ for 14.5 GHz microwaves. The He plasma density is higher for $470 \mathrm{~W}$ of microwave power than at $200 \mathrm{~W}$. From the figure 7 it can be seen that the $1+$ transport efficiency at low injection energies is higher at lower plasma densities and the opposite holds for the higher plasma densities. It implies that the incident $1+$ ions are captured more frequently at high densities than at lower densities. The estimated $\mathrm{O}_{2}$ plasma density at a rf power of $200 \mathrm{~W}$ is in good agreement with the estimates reported in the publication [30]. The estimated He plasma densities at a rf power of $470 \mathrm{~W}$ and $200 \mathrm{~W}$ are close to the estimates reported in [16]. To find out the plausible explanation for the difference in total capture for three cases, the obtained plasma parameters $\left(n_{e}, \mathrm{k} T_{i}\right.$ and $\left.Z_{\text {eff }}\right)$ can be used to investigate the collision rates between the incident $1+$ ions and background plasma ions and mean free paths with respect to cumulative long range ion-ion collisions. The ion-ion collision frequency scales with $n_{e}$ and plasma ion temperature $\left[\nu_{i i} \propto n_{e} /\left(k T_{i}^{3 / 2}\right)\right]$. The mean free path of long range ion-ion collisions [16] of the $1+$ ions $\left(\lambda_{i i}^{1+}\right)$ can be estimated using the ion temperatures and electron densities obtained from the simulations using the relation

$$
\lambda_{i i}^{1+}=-\frac{L}{\frac{L}{\lambda_{1+}}+\ln \left(\frac{I_{\text {plasma }}}{I_{\text {vacuum }}}\right)},
$$

where $L$ is the effective plasma length, $\lambda_{1+}$ is the mean free path of in-flight electron impact ionization, $I_{\text {plasma }}$ is the $1+$ ions transported through the SP1 CB plasma at optimum $\Delta \mathrm{V}$ and $I_{\text {vacuum }}$ is the $1+$ ions transported without SP1 CB plasma. The effective plasma length is approximately $120 \mathrm{~mm}( \pm 10 \%$ depending on the magnetic field configuration applied in the three cases). The electron impact ionization cross sections are calculated from the Lotz fromula [28]. The corresponding $1+$ ions collision 
TABLE VIII. Estimated ion-ion collision frequencies (lower limit) and the $1+$ mean free path (upper limit) for $\mathrm{Na}^{1+}$ and $\mathrm{K}^{1+}$ ions, and the corresponding $\mathrm{He}$ and $\mathrm{O}_{2}$ plasma densities at different microwave powers. The value of Coulomb algorithm is assumed as 10 .

\begin{tabular}{lccc}
\hline \hline & $\mathrm{Na}^{1+}+\mathrm{He}$ & $\mathrm{K}^{1+}+\mathrm{He}$ & $\mathrm{K}^{1+}+\mathrm{O}_{2}$ \\
\hline RF power [W] & 470 & 209 & 207 \\
$n_{e}\left[\times 10^{11} \mathrm{~cm}^{-3}\right]$ & 3.5 & 2.0 & 1.5 \\
$\left(\Delta V_{\exp }-V_{P}\right)$ & 5 & 6.5 & 5.5 \\
$\nu_{i i}^{1+}[\mathrm{KHz}]$ & 182 & 163 & 109 \\
$\lambda_{i i}^{1+}[\mathrm{mm}]$ & 37.08 & 34.63 & 47.52 \\
capture [\%] & 16.44 & 24.52 & 7.26 \\
\hline \hline
\end{tabular}

frequency can be calculated from the estimated mean free path by

$$
\nu_{i i}^{1+}=\frac{v_{a}}{\lambda_{i i}^{1+}},
$$

where $v_{a}$ is the velocity of incident $1+$ ions at an optimum injection energy. Using the above equations, the upper limit of $1+$ ions mean free paths and the lower limits of the ionion collisions frequencies for $\mathrm{Na}^{1+}$ and $\mathrm{K}^{1+}$ in $\mathrm{He}$ and $\mathrm{O}_{2}$ plasma at different microwave power levels are shown in the Table VIII.

It can be seen that the ion-ion collision frequency of $\mathrm{Na}^{1+}$ is bit higher compared to the $\mathrm{K}^{1+}$ ions in He plasma. The estimated ion-ion collision mean free paths of $\mathrm{Na}^{1+}$ and $\mathrm{K}^{1+}$ ions are in a similar range due to the speed of the $1+$ ions. The resulting total capture is slightly higher for $\mathrm{K}^{1+}$ ions compared to $\mathrm{Na}^{1+}$ ions. This is in good agreement with the experimental results, which also revealed higher efficiencies in the case of $\mathrm{K}^{1+}$ compared to $\mathrm{Na}^{1+}$ in $\mathrm{He}$ plasma (see Table I). The difference in total capture between the $\mathrm{Na}^{1+}$ and $\mathrm{K}^{1+}$ ions is perhaps due a better focusing of the $\mathrm{K}^{1+}$ beam in comparison to $\mathrm{Na}^{1+}$ beam after the injection optics (electrostatic triplet) at the injection of SP1 CB.

In the case of $\mathrm{K}^{1+}$ ions in $\mathrm{He}$ and $\mathrm{O}_{2}$ plasma, the calculated mean free path of ion-ion collisions is approximately $30 \%$ longer for $\mathrm{K}^{1+}$ in $\mathrm{O}_{2}$ plasma compared to $\mathrm{K}^{1+}$ ions in He plasma. Correspondingly, the estimated ion-ion collision frequencies are higher for $\mathrm{K}^{1+}$ in He plasma compared to $\mathrm{K}^{1+}$ ions in $\mathrm{O}_{2}$ plasma. The differences in the collision rates can be seen in the experimental results (refer Table I), where the fraction of incident $\mathrm{K}^{1+}$ ions propagating through the $\mathrm{O}_{2}$ plasma is approximately $6.5 \%$, while the $\mathrm{K}^{1+}$ ions propagating through the He plasma is around $2.5 \%$ at equivalent microwave powers. The good agreement between the experimental and simulated trends indicates that the difference in total capture between $\mathrm{K}^{1+}$ ions in $\mathrm{O}_{2}$ and $\mathrm{He}$ plasma is due to the difference in the collisional rates.
TABLE IX. Comparison of optimum 1+ injection energies from theoretical calculations, experiments and MCBC simulations.

\begin{tabular}{lccc}
\hline \hline & $\mathrm{Na}^{1+}+\mathrm{He}$ & $\mathrm{K}^{1+}+\mathrm{He}$ & $\mathrm{K}^{1+}+\mathrm{O}_{2}$ \\
\hline$V_{p}[\mathrm{~V}]$ & 1.5 & 1 & 1 \\
$E_{a}[\mathrm{eV}]$ & $5.17-6.32$ & $6.8-7.8$ & $2.43-6.09$ \\
$E_{\mathrm{inj}}^{\exp }[\mathrm{eV}]$ & 5 & 6.5 & 5.5 \\
$E_{\mathrm{inj}}^{\operatorname{sim}}[\mathrm{eV}]$ & 6 & 7.5 & 7.5 \\
\hline \hline
\end{tabular}

Coming back to the beam slowing down theory (see Sec. I), the optimum capture is achieved when the ratio of longitudinal component of the injected $1+$ ion velocity $\left(v_{a}\right)$ to the thermal velocity of the plasma ions $\left(v_{b}\right)$ is 1 [15]. Subtracting the plasma potential obtained from the simulation of three cases, optimum injection energies from theoretical calculations, simulations and experiments are presented in Table IX. It is clear that the optimum injection energies obtained from experiments $\left(E_{\text {inj }}^{\exp }\right)$ and simulations $\left(E_{\mathrm{inj}}^{\mathrm{sim}}\right)$ lies within the theoretical value $\left(E_{a}\right)$. In the case $\mathrm{K}^{1+}+\mathrm{O}_{2}$, the simulated optimum injection energy is slightly higher than the theoretical and experimental one. The plasma potential was found to be very low for the three cases (up to few volts). This is may be due to the grounded tube positioned close to the SP1 CB plasma chamber especially in the $\mathrm{K}^{1+}+\mathrm{O}_{2}$ experiment. With this kind of configuration, the decelerating field created by the grounded tube might push the cold electrons toward the dense plasma region, resulting to a very low potential near the entrance of the SP1 CB. The effect of grounded tube on the plasma potential should be studied intensively with different plasma species and axial positions of the grounded tube.

Finally, many simulations were performed to understand the influence of $1+$ beam emittance and injection optics (electrostatic triplet) on the capture dynamics. One of the experimental cases (e.g., $\mathrm{Na}^{1+}+\mathrm{He}$ ) was considered to investigate the influence of injected beam emittance (beam radius and divergence) on the beam capture dynamics. $1+$ beam emittance sweeps were performed by scaling the beam properties of $50 \pi \mathrm{mm} . \mathrm{mrad}$ emittance. For each emittance value, the total capture and the particle losses were recorded at an optimum $\Delta \mathrm{V}$ of $7.5 \mathrm{eV}$. Figure 14 presents the summary of total $1+$ ion capture and ion losses obtained during the $1+$ beam emittance study. At smaller $1+$ beam emittance $(10 \pi . \mathrm{mm} . \mathrm{mrad})$, up to $30 \%$ of $1+$ ions are trapped inside the ECR volume (plasmoid) and a fraction of ions are lost at the injection, chamber wall and toward the plasma electrode. At higher injection emittance (80 $\pi$.mm.mrad), majority of ions are lost near the injection and the total capture is on the order of $3 \%$. The simulation clearly shows that the $1+$ beam emittance 


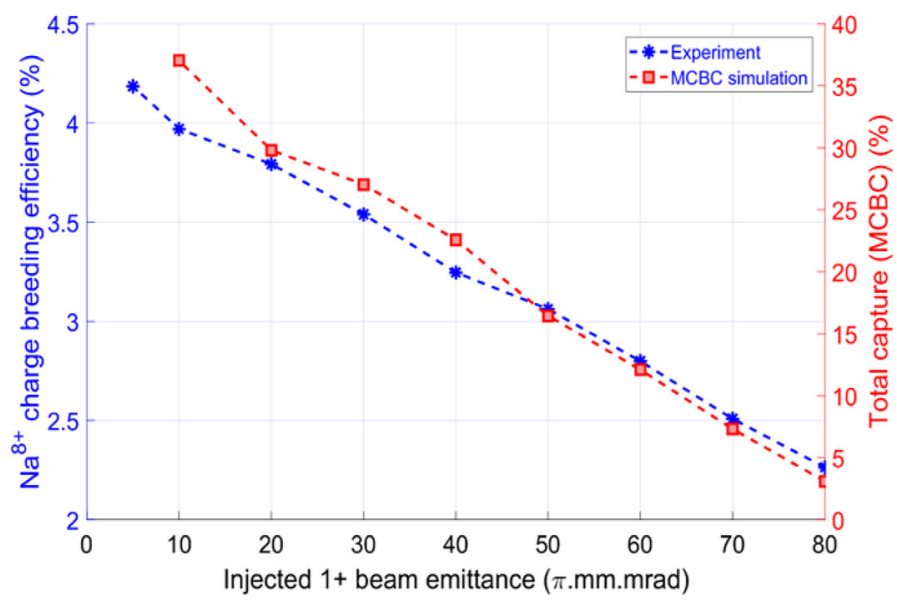

(a)

(A) $\mathrm{Na}^{+}$beam (@20 keV)

\section{Before ES triplet}

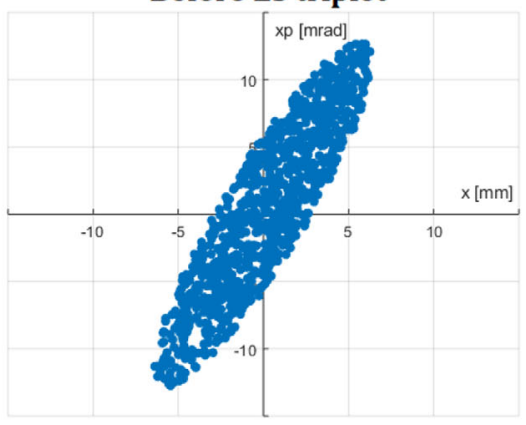

(B) $\mathrm{K}^{+}$beam (@15 keV)

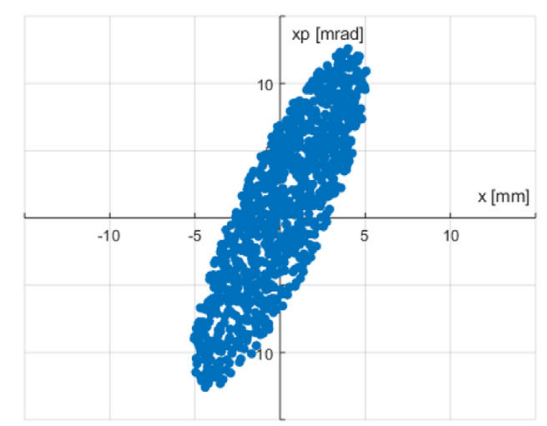

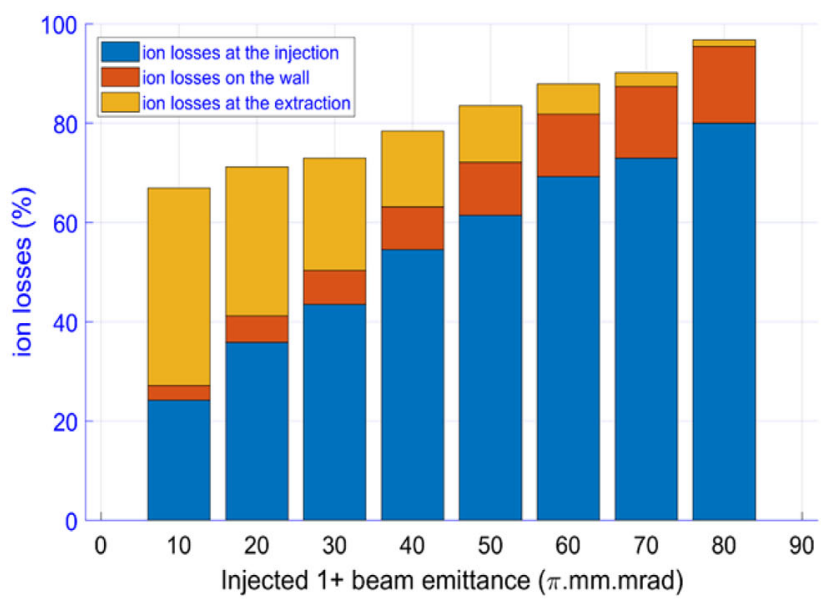

(b)

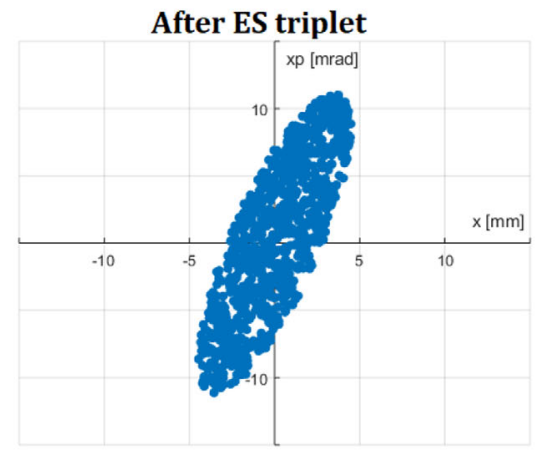

(c)

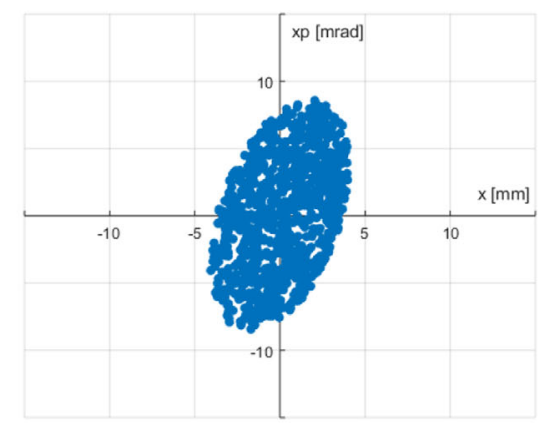

FIG. 14. (a) Comparison between the experimental $\mathrm{Na}^{8+}$ charge breeding efficiency and the total $1+$ ion capture from MCBC. (b) Total number of $1+$ ions lost in the simulation domain (reflected back, lost to the wall, and lost toward the extraction). (c) Simulated transverse beam positions of $20 \mathrm{keV} \mathrm{Na}^{1+}$ beam (top) and $15 \mathrm{keV} \mathrm{K}^{1+}$ beam (bottom) before and after the injection optics (electrostatic triplet).

(radius and divergence) should be as small as possible to achieve high injection and capture efficiency and consequently, high charge breeding efficiency. Comparing the simulation and experimental results, it can be said that a similar behavior takes place in reality.

In simulations, $\mathrm{Na}^{1+}$ and $\mathrm{K}^{1+}$ beams with an emittance of $50 \pi$.mm.mrad were used to reproduce the experimental $\Delta \mathrm{V}$ curves and achieved a total capture of $16 \%$ and $24 \%$ with $\mathrm{He}$ as support gas. The beam characteristics for the two cases were observed to be slightly different at the slit position S13 shown in Fig. 1. Moreover, the potentials applied to the injection optics were different in the two cases. The simulated transverse beam positions at the initial point and after the injection electrostatic triplet are compared and presented in the Fig. 14. It is clear that the $\mathrm{K}^{1+}$ beam (at $15 \mathrm{keV}$ ) is better focused after passing through the electrostatic triplet which can explain the difference in efficiencies. 


\section{CONCLUSION}

The presented numerical simulations using $\mathrm{MCBC}$ have demonstrated that the incident $1+$ beam can be applied as a probe to understand the role of Coulomb collisions in slowing down and capture process. The Coulomb collisions in the MCBC were modeled from the formulations derived by Boozer and treated as a continuous force. The model was further improved by implementing a simplified ECR plasma with a potential dip structure to confine the ions. Applying the presented simulation strategy and a precise combination of plasma parameters, $\mathrm{MCBC}$ can reproduce the trends of complete $\Delta \mathrm{V}$ curves (from low to very high injection energies) measured during the experiments. The optimum plasma parameters and their error bars were estimated by applying the $\chi^{2}$ technique. As such, the quantitative analysis presented here gives results mostly in agreement but is also going one step further than the more qualitative analysis done in [35] and [37]. The relative error bars give a quantitative information on the most critical parameters for the stopping, capture and in-flight ionization of $1+$ beams, namely the $\mathrm{k} T_{i}, n_{e}$, and $T_{e}$. Transmission/in-flight ionization curves for $1+$ and $2+$ charge states are reproduced on a wider range so that normalization effects for large injection energies-corresponding asymptotically to transport efficiency for the $1+$ beam and in-flight ionization for the $2+$ beam respectively - are duly taken into account. The agreement between the experimental results and the simulated curves is remarkable and this for different systems, giving a good confidence in the modeling of the Coulomb interaction used by MCBC. Also the simplified plasma model seems sufficiently accurate to describe the experimental results.

The ion temperatures and electron densities at different rf powers are in good agreement with the estimates reported in $[16,30,42]$. The simulation results and analysis indicate that the ECR charge breeder plasma has to be tuned at relatively low density (an order of magnitude below the critical density for $14.5 \mathrm{GHz}$ ) and low ion temperature which are critical paramaters for the thermalization of ions. In addition, charge breeding efficiency results tell us that a low emittance $1+$ beam is necessary to increase injection as well as capture efficiency and consequently, the charge breeding efficiency. Therefore, a RFQ beam cooler is being proposed at the injection of the charge breeder. It will also allow to decrease the aperture of the soft iron at the injection leading to an increase in the axial magnetic field at injection and consequently, improving the charge breeding performances.

A good agreement between the simulations and experiments indicate that the plasma parameters extracted from MCBC can be used to investigate the charge breeding performances. Along this line, a batch model [43] using these parameters was lately developed to reproduce the charge state distribution evolution from the SP1 charge breeder. Preliminary results shown in [44] will be developed in a forthcoming article. The parameters obtained from MCBC will also be useful for a consistency check of results with our being-developed particle in cell (PIC) code [45]. This code will enable further investigations of the $1+$ beam capture, including ingredients presently missing in MCBC. Among others, it should enable the simulation of a continuous injection of $1+$ ions, the presheath effect and dynamics of plasma particles in electromagnetic fields.

In this perspective, the simulations will be extended to lower and heavier mass ions $\left(\mathrm{Na}^{+}, \mathrm{K}^{+}, \mathrm{Rb}^{+}\right.$and $\left.\mathrm{Cs}^{+}\right)$into hydrogen plasma. The parameters obtained from these simulations will permit to study the charge breeding performances. Simulations also evidenced the temperature of background plasma ions that had to be adjusted by parameters such as the rf power to reach an optimum injection energy in the range of 6 to $7.5 \mathrm{eV}$ whatever the buffer gas/1+ ions injected system considered in our charge breeder, which is a peculiar fact still to be elucidated. Finally, the PIC code will allow to study the impact of inner plasma ion properties during the interaction of incident $1+$ beam with an ECR charge breeder plasma and can provide an important benchmarking of simulation results and model assumptions encoded in the simulations.

[1] P. Van Duppen, Isotope separation on line and post acceleration, in The Euroschool Lectures on Physics with Exotic Beams, Vol. II, edited by J. Al-Khalili and E. Roeckl (Springer Berlin Heidelberg, Berlin, Heidelberg, 2006), pp. 37-77.

[2] O. Kamalou, J. Angot, O. Bajeat, F. Chautard, P. Delahaye, M. Dubois, T. Lamy, L. Maunoury, G. Normand, and A. Savalle, in 13th International Conference on Heavy Ion Accelerator Technology (HIAT 2015), Yokohama, Japan (2016) p. MOA1C02, http://hal.in2p3.fr/in2p3-01196241.

[3] A. Villari, C. Barué, G. Gaubert, S. Gibouin, Y. Huguet, P. Jardin, S. Kandri-Rody, F. Pellemoine, N. Lecesne, R. Leroy, M. Lewitowicz, and C. Marry, Nucl. Phys. A701, 476 (2002).

[4] L. Maunoury, Production de faisceaux d'ions radioactifs multicharges pour SPIRAL: Etudes et realisation du premier ensemble cible-source, theses, Université de Caen, 1998.

[5] P. Jardin, O. Bajeat, C. Couratin, P. Delahaye, M. Dubois, C. Feierstein, F. Pellemoine, P. Lecomte, P. Lehérissier, L. Maunoury, M. Saint-Laurent, and E. Traykov, Rev. Sci. Instrum. 83, 02A911 (2012).

[6] P. Delahaye et al., in 18th International Conference on Electromagnetic Isotope Separators and Related Topics (JACoW, Geneva, Switzerland, 2018), Vol. 463, pp. 339-344.

[7] L. Penescu, R. Catherall, J. Lettry, and T. Stora, Rev. Sci. Instrum. 81, 02A906 (2010).

[8] P. Chauveau, P. Delahaye, M. Babo, H. Bouzomita, O. Bajeat, M. Dubois, R. Frigot, G. Grinyer, J. Grinyer, P. Jardin, C. Leboucher, L. Maunoury, C. Seiffert, T. Stora, 
J. Thomas, and E. Traykov, Nucl. Instrum. Methods Phys. Res., Sect. B 376, 35 (2016).

[9] L. Boy, Problèmes posés par l'accélération d'ions radioactifs dans le projet SPIRAL. Réglage et stabilisation de l'accélérateur, theses, Université Pierre et Marie CurieParis VI, 1997.

[10] C. Tamburella, Projet PIAFE: production d'etats de charges elevees pour des ions radioactifs, theses, Université Joseph-Fourier-Grenoble I, 1996.

[11] T. Lamy, J.-F. Bruandet, N. Chauvin, J.-C. Curdy, M. Fruneau, R. Geller, G. Gimond, P. Sole, J.-L. VieuxRochas, G. Gaubert, L. Maunoury, P. Sortais, and A. C. C. Villari, Rev. Sci. Instrum. 69, 741 (1998).

[12] P. Delahaye, O. Kester, C. Barton, T. Lamy, M. MarieJeanne, and F. Wenander, Eur. Phys. J. A 46, 421 (2010).

[13] F. Wenander, Nucl. Phys. A746, 40 (2004).

[14] T. Lamy, J. Curdy, R. Geller, C. Peaucelle, P. Sole, P. Sortais, T. Thuillier, D. Voulot, K. Jayamanna, M. Olivo, P. Schmor, and D. Yuan, in European Particle Accelerator Conference 8 EPAC 2002, edited by G. T. et al. (CERN, Paris, France, 2002), pp. 1724-1726.

[15] J. L. Delcroix and A. Bers, Physique des Plasmas (EDP Sciences, Paris, France, 1994).

[16] O. Tarvainen, H. Koivisto, A. Galatà, J. Angot, T. Lamy, T. Thuillier, P. Delahaye, L. Maunoury, D. Mascali, and L. Neri, Phys. Rev. Accel. Beams 19, 053402 (2016).

[17] R. Geller, T. Lamy, and P. Sortais, Rev. Sci. Instrum. 77, 03B107 (2006).

[18] L. Maunoury, P. Delahaye, M. Dubois, J. Angot, P. Sole, O. Bajeat, C. Barton, R. Frigot, A. Jeanne, P. Jardin, O. Kamalou, P. Lecomte, B. Osmond, G. Peschard, T. Lamy, and A. Savalle, Rev. Sci. Instrum. 87, $02 B 508$ (2016).

[19] J.-S. Kim, L. Zhao, B. Cluggish, and R. Pardo, Rev. Sci. Instrum. 78, 103503 (2007).

[20] L. Zhao, B. Cluggish, J. Kim, R. Pardo, and R. Vondrasek, Rev. Sci. Instrum. 81, 02 A304 (2010).

[21] T. Lamy, J. Angot, T. Thuillier, P. Delahaye, L. Manoury, A. Galatà, G. Patti, H. Koivisto, O. Tarvainen, J. Choinski, and L. Standylo, 21st International Workshop on ECR Ion Sources (ECRIS 2014), Nizhny Novgorod, Russia (2014), pp. 120-126, http://hal.in2p3.fr/in2p3-01058052.

[22] B. A. Trubnikov, Rev. Plasma Phys. 1, 105 (1965).

[23] J. D. Huba, Plasma Physics (Naval Research Laboratory, Washington, DC, 2013), pp. 1-71.

[24] S. Chandrasekhar, Astrophys. J. 93, 285 (1941).
[25] S. Chandrasekhar, Astrophys. J. 97, 255 (1943).

[26] A. H. Boozer and G. Kuo-Petravic, Phys. Fluids 24, 851 (1981).

[27] A. H. Boozer, Phys. Plasmas 9, 4389 (2002).

[28] W. Lotz, Astrophys. J. Suppl. Ser. 14, 207 (1967).

[29] A. Müller and E. Salzborn, Phys. Lett. A 62, 391 (1977).

[30] O. Tarvainen, T. Lamy, J. Angot, T. Thuillier, P. Delahaye, L. Maunoury, J. Choinski, L. Standylo, A. Galatà, G. Patti, and H. Koivisto, Plasma Sources Sci. Technol. 24 (2015).

[31] D. A. Dahl, Int. J. Mass Spectrom. 200, 3 (2000).

[32] A. A. Ivanov and K. Wiesemann, IEEE Trans. Plasma Sci. 33, 1743 (2005).

[33] D. Mascali, S. Gammino, L. Celona, and G. Ciavola, Rev. Sci. Instrum. 83, 02A336 (2012).

[34] A. Galatà, D. Mascali, L. Neri, and L. Celona, Plasma Sources Sci. Technol. 25 (2016).

[35] V. Mironov, S. Bogomolov, A. Bondarchenko, A. Efremov, and V. Loginov, AIP Conf. Proc. 2011, 070003 (2018), arXiv:1706.07591.

[36] G. Shirkov, Rev. Sci. Instrum. 71, 850 (2000).

[37] W. G. Cochran, Ann. Math. Stat. 23, 315 (1952).

[38] M. Sakieldien, Characteristic K $\alpha$ emission of electron cyclotron resonance ion source plasmas, Ph.D. thesis, University of Jyväskylä, 2018.

[39] S. Gammino, CAS-CERN Accelerator School: Ion Sources, Senec, Slovakia, 2012, edited by R. Bailey (2014), https://doi.org/10.5170/CERN-2013-007.123.

[40] A. Annaluru, Beam optics transport and fundamental processes involving a charge breeder in the upgraded SPIRAL1 facility, Ph.D. thesis, Université de Caen, 2019.

[41] V. Mironov, B. Sergey, A. Bondarchenko, A. Efremov, and V. Loginov, Phys. Rev. Accel. Beams 18 (2015).

[42] A. Girard, A. Drentje, G. Melin, and D. Hitz, J. Appl. Phys. 86, 4772 (1999).

[43] R. Geller, Annu. Rev. Nucl. Part. Sci. 40, 15 (1990).

[44] P. Delahaye, Capture, ionisation et piégeage d'isotopes pour la physique fondamentale (2020), http://hal.in2p3.fr/ tel-03101427.

[45] A. Leduc, L. Maunoury, and T. Thuillier, in 23rd International Workshop on ECR Ion Sources, Catania, Italy (JACoW Publishing, Geneva, Switzerland, 2018), p. WEB2, http://jacow.org/ecris2018/papers/web2.pdf. 